\title{
Energy-Efficient Clustering Design for M2M Communications
}

\author{
PENG ZHANG
}

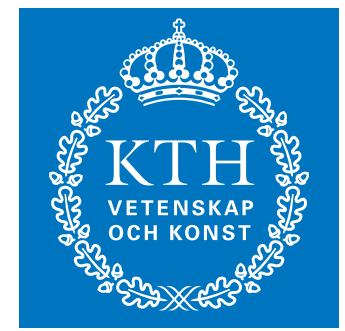

KTH Information and Communication Technology 
Royal Institute of Technology

\section{Abstract}

Wireless Systems

School of Electrical Engineering

Master of Science

\section{Energy-Efficient Clustering Design for M2M Communications}

by Peng Zhang

Machine-to-machine (M2M) communications have appeared as an advanced technology for next-generation communications and are undergoing rapid development. In this project, we investigate M2M communications in a wireless cellular network. In M2M communications, clustering is a technology for more efficient data gathering and higher network energy efficiency. We will analyze existing clustering designs in literature and propose two new clustering designs for M2M communications in cellular networks. Performance of the proposed designs will be evaluated thoroughly using both analytical and simulation tools across many aspects, including energy consumption, dead device ratio, residual energy, and network life. The results show that with simple static energyefficient clustering operations, the network life can be extended by about $50 \%$. 


\section{Acknowledgements}

Firstly of all, I would like to give my special thanks to my parents whose strong support enabled me to complete my study. Secondly, I would like to extend my immense gratitude to Dr Miao Guowang for giving me this precious chance to perform my Degree work in his help and for all his professional supervision and advices. Thirdly, I want to thank all my talent friends: Wang Zhao, Shi Guodong, Zheng ZhiHao, Yang Yanpeng, Mao Mao, XiaoHang Chan and Javier Mendonca Costa. Thanks for their tremendous support during this entire period and for sharing their humongous academic knowledge selflessly. Last but not least I want to thank my girlfriend Xu Lidi. She is smart, lovely and optimistic, who made my days filled with joy. Thanks my small girl! 


\section{Contents}

Abstract $\quad$ i

$\begin{array}{ll}\text { Acknowledgements } & \text { ii }\end{array}$

Contents

List of Figures $\quad$ v

List of Tables vi

Abbreviations vii

1 Introduction $\quad 1$

1.1 M2M Communications . . . . . . . . . . . . . . . . . 1

1.2 Energy Efficiency . . . . . . . . . . . . . . . . . . . . 3

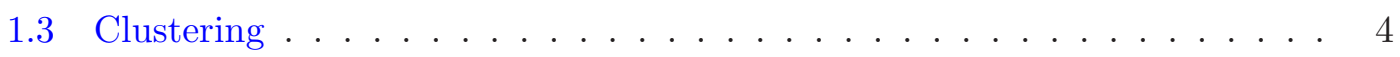

1.4 Thesis Structure . . . . . . . . . . . . . . . 6

$\begin{array}{llr}2 & \text { Objective and Methodology } & 7\end{array}$

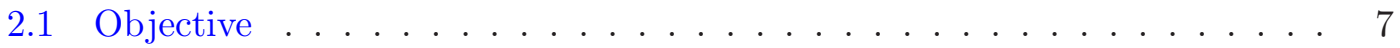

2.2 Methodology . . . . . . . . . . . . . . . . 8

3 System Model $\quad 10$

3.1 Assumption . . . . . . . . . . . . . . . . . . . . 10

3.2 Building Energy Efficiency Mathematical Model . . . . . . . . . . . . . . . 11

3.3 Mathematical Derivation . . . . . . . . . . . . . . . . . . 12

3.4 Expectation for Path loss and Cluster Size . . . . . . . . . . . . . . . 14

3.5 Optimal Value Derivation . . . . . . . . . . . . . . . . . . 17

4 Static Cluster Design $\quad 20$

4.1 Deploy Devices . . . . . . . . . . . . . . . . . . . . . . 21

4.2 The Number of Cluster Heads . . . . . . . . . . . . . . . . . . . . . 21

4.3 Cell Segmentation . . . . . . . . . . . . . . . . . . . . . . . . . . . . . . . . . . . 22

4.4 Cluster Head Selection . . . . . . . . . . . . . . . . . . . 23

4.4 .1 Inter Communication Cost . . . . . . . . . . . . . 24

4.4 .2 Intra Communication Cost . . . . . . . . . . . . . . . . 24

4.4 .3 Cost Function . . . . . . . . . . . . . . . . . . 25 
4.5 Cluster Head Reselection _. . . . . . . . . . . . . . . . . 27

4.5.1 Energy Consumption for Device . . . . . . . . . . . . 27

4.5.2 Cluster Head Reselection . . . . . . . . . . . . . . . . 28

5 Performance Analysis for Static Cluster Design 29

5.1 Residual Energy Analysis . . . . . . . . . . . . . . . . . . . . 29

5.2 Dead Devices and Lifetime Analysis . . . . . . . . . . . . . . . 32

6 Dynamic Cluster Design 36

6.1 Cluster Form . . . . . . . . . . . . . . . . . . 37

6.2 Cluster Reselection . . . . . . . . . . . . . . . . . . . . . 40

6.3 Result Comparing . . . . . . . . . . . . . . . . . . . 41

7 Conclusion and Further Works $\quad 46$ 


\section{List of Figures}

1.1 A general model of the smart grid. . . . . . . . . . . . . . . . 2

3.1 Transmit power for devices. . . . . . . . . . . . . . . . . . 12

3.2 Energy efficiency with different $p$ and $\beta \ldots \ldots \ldots \ldots$

4.1 The flow chart for static cluster design. . . . . . . . . . . . . 20

4.2 The relation between optimal $p$ and $\beta$ in static design. . . . . . . . . . 22

4.3 Segmentation in first quadrant. . . . . . . . . . . . . . 23

4.4 Intra consumption. . . . . . . . . . . . . . . . . . 25

4.5 Cluster formation. . . . . . . . . . . . . . . . . . . 26

5.1 Whole system residual energy for different $p$ in 30 days. . . . . . . . . . 30

5.2 Residual energy for different $p$ on $100^{t h}, 200^{t h}, 300^{t h}$ day. . . . . . . . . . 31

5.3 Dead devices with different $p \ldots \ldots \ldots \ldots \ldots$. . . . . . . . . 34

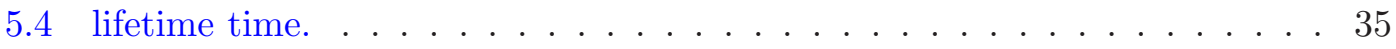

6.1 The flow chart for dynamic design. . . . . . . . . . . . . . . 37

6.2 Optimal $p$ as different number in dynamic design. . . . . . . . . . . . . 39

6.3 Optimal $p$ as different number by using dynamic and static design. . . . . 42

6.4 Residual energy comparing for different $n$ in 30 days . . . . . . . . . . . 43

6.5 Residual energy comparing for different $n$ on $100^{\text {th }}, 150^{\text {th }}, 250^{\text {th }}, 300^{\text {th }}$ day. 44

6.6 Dead devices for two ways with $n=500 \ldots \ldots \ldots \ldots . \ldots . \ldots 45$

6.7 Dead devices for two ways with $n=6000 \ldots \ldots \ldots \ldots$ 


\section{List of Tables}

1.1 Comparison of the presented clustering algorithm for WSNs . . . . . . 6

3.1 System parameters $[1-4] \quad \ldots \ldots \ldots \ldots \ldots \ldots$

5.1 System residual energy on different days . . . . . . . . . . . . . . . 32 


\title{
Abbreviations
}

\author{
M2M Machine to Machine \\ H2H Human to Human \\ EE Energy Efficiency \\ QoS Quality-of-Service \\ OFDMA Orthogonal Frequency Division Multiple Access \\ BS Base Station \\ CH Cluster Head \\ GM Group Member \\ WSN Wireless Sensor Network \\ SP Short Distance Propagation \\ LP Long Distance Propagation \\ LTE Long Term Evolution \\ 3GPP Third Generation Partnership Project
}




\section{Chapter 1}

\section{Introduction}

One of the most significant current discussions in wireless communication is the diversity on applications using cellular networks. In most of these existing applications, Machine to Machine (M2M) communications has been regarded as a new type of communication, which is a rapidly developing technology for huge scalable networks of wireless devices independent on human interaction [5]. In the following section we introduce a background of M2M communications.

\subsection{M2M Communications}

M2M communications means that by utilizing digital communication technologies, millions of different M2M devices (such as wireless sensors or meters) connect each other directly. M2M communications have been discussed as the next technology revolution after the computer and the internet [6]. There are both technical and economic drivers for the industry to continue to develop M2M communications. The economic drivers include that the traditional human-based market is saturated in developed countries and technical progresses in the industry extends the coverage of wireless networks and at the same time lowering the costs per bit [7]. Some reports from companies have show the huge potential for this market, with millions of M2M devices being networked over the next 5 years and revenues exceed 300 billion USD (Harbor Research 2009).

In the technical aspect of M2M communications a group of devices gather data and forward it to a server, being routed over the wireless networks and internet to the 
servers. For example, a power control system in the city center sends commands to user devices which issue the electrical signals to make machines take action, and M2M communications have an immense potential for future applications of this technology. Because of these economic and technical potential, the IEEE and 3GPP organisms work together to identify the related issues.

There is no standard definition for M2M devices for determining their application categories. In [1], it turns out that Smart metering, tele-health and asset tracking might represent the main traffic sources when it comes to M2M communications.

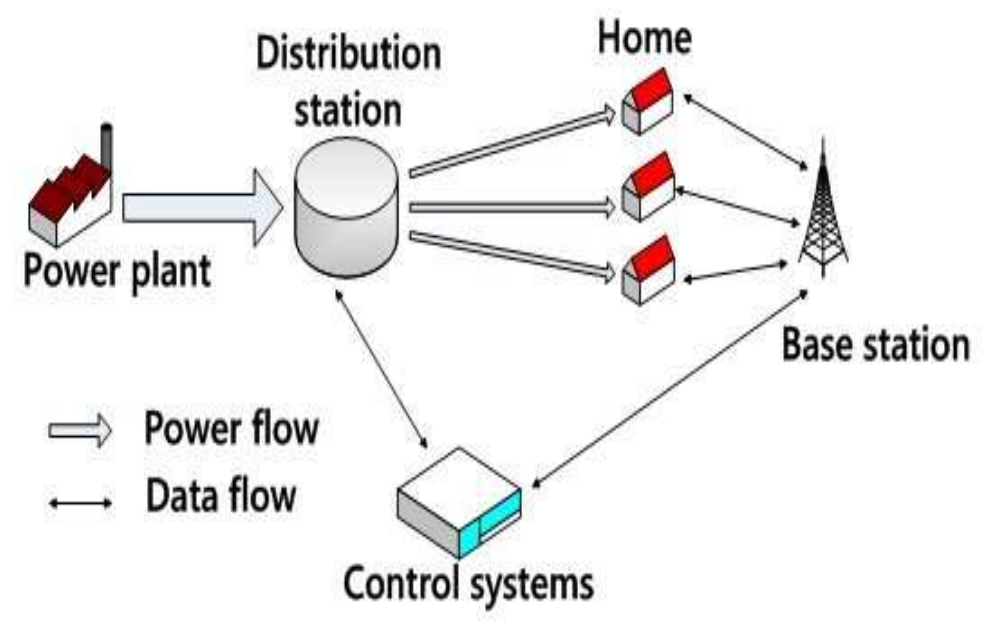

Figure 1.1: A general model of the smart grid.

Figure 1.1 describes a general model of the smart grid which is one of smart metering applications. Smart Metering is utilized for remote monitoring and energy application by recording the consumption of electric energy. It provides a two-way communication between the meter and system. It can be used for monitoring the record for power, gas, water, heating, grid control or industrial metering [8].

Remote monitoring of patients is used in tele-health applications. As an example, when serious body conditions are detected in a patient an alarm signal needs to be sent to the hospital. In order to accomplish that goal, a body area network (BAN) of sensors is deployed around a patient to monitor health indicators such as blood pressure, body temperature and heart rate [9].

In the asset tracking application sensor devices monitor objects and help companies to keep track of their valuable assets, moreover to manage their routing. In general, 
assets are divided into fixed assets like vending machines and mobile assets like cargo transportation [10].

When we want to find a good wireless network for M2M communications, cellular networks with the advantages of low cost, high data rate and large coverage which have been considered as one of the best choices [11]. There are some typical characteristics for M2M communications, such as small payload, little traffic per device and massive number of devices $[12,13]$. Cellular networks, which are mainly designed for mobile phone communications, are already being used for some M2M applications nowadays. In comparison to human-to-human communications, there are many incompatible factors that we should consider, being the Energy Efficiency (EE) one of the big issues.

\section{$1.2 \quad$ Energy Efficiency}

The main goal is to reduce the amount of energy required by the machines to provide their services. In the wireless communication area, we want to reduce the energy consumption of the devices while meeting high quality-of-service (QoS) requirements [14]. Considering the ecological benefits and economic profits, not only from operator's perspective but also from users' perspective, energy-efficient wireless communications are necessary.

An appropriate energy efficiency metric is the key for designing the energy-efficient wireless network, because it is directly related to the optimal design. Different EE metrics have been used in previous research studies, being 'bits-per-Joule' one of most popular ones, which is defined as the whole system throughput per unit energy consumption, such as in [15], 'bits-per-Joule' are used for designing uplink OFDMA systems. There are two types of energy consumption introduced in [16]. One of them is know as the transmitting energy, in the front-end amplifier that provides the power for the actual radio frequency transmission. Another is processing energy which handles the consumption of encoding, modulation and other signal processing functions.

In real M2M communications, the majority of the devices work based on battery powered conditions and recharging or replacement of the batteries is not frequent or even impossible in some cases. So we can see how important is to have low energy consumption in M2M communications. The system network lifetime can be defined as most of 'dead' 
devices (such as $90 \%$ devices 'died'), where 'dead' means that the battery of device was exhausted. In our project, the energy consumption for M2M devices include

- Transmitting consumption: The device send the data to neighboring devices or Base Station (BS).

- Electronic circuits: Average energy consumption of device electronics.

- Wake consumption: The consumption for device wake up.

- Sleep consumption: The consumption for device sleep.

In order to make the whole system last as long as possible, an energy-efficient design for M2M communications is necessary and reasonable. The goal is to achieve high energy efficiency, reducing the amount of energy consumed by the devices while guaranteeing the whole system throughput as much as possible. Clustering have been proven to be an energy-efficient way for massive devices communications [17], since it is an effective approach to provide better data aggregation and scalability for large number of devices, conserving the limited energy resources of the devices. In the following section we will study the background of clustering.

\subsection{Clustering}

In a large M2M communication system, if every device communicates to the BS directly, great data congestion and collisions will occur. This will waste power and drain the energy from the devices quickly. Clustering is a good method to overcome these weaknesses. In clustered networks, some devices are selected as cluster heads $(\mathrm{CH})$ for each cluster group, other normal devices in each cluster group transmit their data to the respective $\mathrm{CH}$. The $\mathrm{CH}$ aggregates the data and forwards it to the BS. Clustering increases the efficiency of data transmission by reducing the number of devices attempting to access the BS. Thus, it leads to reduced signal overheads among the network and save the energy of the whole system.

Wireless sensor networks (WSNs) have similar characteristic as M2M communications, such as large number of sensors and battery-powered devices. In the WSNs field, some mature technologies for cluster design are already deployed. 
Energy Efficient Hierarchical Clustering (EEHC) [18]: EEHC is a distributed, randomized clustering algorithm for WSNs. First it utilizes a mathematical derivation to find the optimal value of the parameters $p$ (the probability of each sensor becoming a $\mathrm{CH}$ ) and $k$ (the sensors are no more than $k$ hops away from $\mathrm{CH}$ ), which would ensure minimization of energy consumption. The key point of the derivation of the optimal parameter values is to derive a function for energy used in the network to transmit data to the BS and find the values that would minimize it. Each sensor advertises itself as a $\mathrm{CH}$ (volunteer cluster head) with probability $p$ to the sensors within its radio range. All sensors within $k$ hops range of a $\mathrm{CH}$ receive this advertisement either by direct communication or by forwarding. Any sensors that receive this advertisement and is not itself a $\mathrm{CH}$ becomes the member of the closest cluster. EEHC is a multi-hop way cluster algorithm, its energy consumption function do not consider the wireless propagation model. It also do not mention the $\mathrm{CHs}$ reselection, it is obvious that the battery of $\mathrm{CH}$ will be exhausted very fast.

Hybrid Energy Efficient Distributed Clustering (HEED) [19]: HEED is a multi-hop clustering algorithm for WSNs. There are two key parameters which are used to select $\mathrm{CHs}$, one is intra-cluster communication cost and another is residual energy. Intracluster communication cost is a function that reflects the cluster size and the sensor's proximity to the neighboring sensor. Residual energy is used to ensure that sensor having a high residual energy can become CH. HEED considers a hybrid of energy and communication cost when selecting $\mathrm{CHs}$, it provides a uniform $\mathrm{CH}$ distribution and doer not select $\mathrm{CHs}$ randomly. HEED considers the $\mathrm{CH}$ s reselection, but his intra-cluster communication function do not mention propagation model.

Low Energy Adaptive clustering Hierarchy (LEACH) [20]: LEACH forms clusters based on the received signal strength. First, a sensor decides to be a $\mathrm{CH}$ with a probability $p$ and broadcasts its decision. Each non-CH sensor determines its cluster group by choosing the $\mathrm{CH}$ that can be reached using the least communication energy. The $\mathrm{CH}$ is rotated periodically among the all sensors of the cluster in order to balance the load. LEACH forms one-hop intra and inter cluster topology where each sensor can transmit directly to the $\mathrm{CH}$ and BS. The propagation model used in LEACH is free space model, it is not applicable to networks deployed in large region. 
Table 1.1 compares the algorithm discuss in this part. In here, SP means short distance propagation and LP means long distance propagation.

TABLE 1.1: Comparison of the presented clustering algorithm for WSNs

\begin{tabular}{|c|c|c|c|c|c|}
\hline $\begin{array}{c}\text { Clustering } \\
\text { algorithm }\end{array}$ & $\begin{array}{c}\text { Intra } \\
\text { cluster }\end{array}$ & $\begin{array}{c}\text { Inter } \\
\text { cluster }\end{array}$ & $\begin{array}{c}\mathrm{CH} \\
\text { selection }\end{array}$ & $\begin{array}{c}\mathrm{CH} \\
\text { reselection }\end{array}$ & $\begin{array}{c}\text { Propagation } \\
\text { model }\end{array}$ \\
\hline EEHC & M-hop & M-hop & Random & No & No \\
HEED & 1-hop & M-hop & Random & Yes & No \\
LEACH & 1-hop & Direct & Random & Yes & SP \\
Our Design & 1-hop & Direct & Cost & Yes & LP \& SP \\
\hline
\end{tabular}

\subsection{Thesis Structure}

The report is divided to seven Chapters as follows: in Chapter 2, the goal and methodology of this project are introduced. Chapter 3 gives a detailed description for building mathematical model. The static cluster design and its performance are explained in Chapter 4 and 5. In Chapter 6, the description for dynamic cluster design and the comparison between these two design are presented. Finally, the conclusions are drawn and the future work is given in the last chapter. 


\section{Chapter 2}

\section{Objective and Methodology}

From the introduction, we have realized that there is a huge number of M2M devices in one cell, but LTE was first developed to support Human to Human $(\mathrm{H} 2 \mathrm{H})$ traffic with requirements such as high data rates. This change in traffic characteristics from $\mathrm{H} 2 \mathrm{H}$ to M2M generates problems for the network [21]. LTE offers both extensive coverage and large capacity. However, for low data rates and massive density of devices, the capacity may be limited by the control channels which become the main bottleneck for M2M communications over LTE. When this massive amount of devices attempts to connect with network congestion will occur and energy will be wasted.

If every device connects to the base station directly it means that almost every device will use long distance propagation model for transmitting their data to the BS. Thus, the energy consumption will be huge. Cluster design is a good way to save the system energy. Some devices send their data to a cluster head and this one forwards the data to the BS. The energy consumption of communication in the cluster (intra communication) will be less, since the devices are close to each other and short distance propagation will be utilized.

\section{$2.1 \quad$ Objective}

For a fixed system bandwidth $\left(w_{1}\right)$ and fixed bandwidth $\left(w_{2}\right)$ in a cluster, more number of cluster heads will lead to lower data rates for the cluster heads, meaning that the cluster heads will use more time to transmit data and also, the wake time will be longer. 
On the other hand, fewer number of cluster heads will increase the energy consumption for the intra communication, the average distance between cluster heads and the group members will increase while decreasing the data rates for the group members. So the key point for us is how to obtain an appropriate amount for clusters. Based on two different ways that decide the number of cluster heads, we create two cluster design algorithms. One of them is a static cluster design and another is dynamic cluster design. We will give a detailed description of them in the next chapters. By using both designs, we want to achieve following objectives:

1. Obtain an optimal number of cluster heads to improve the system energy efficiency.

2. The selected CHs should be well distributed.

3. Find whether the cluster head device selections are reasonable or not.

4. Consider a propagation model for intra cluster communication(GM to $\mathrm{CH})$ and inter cluster communication ( $\mathrm{CH}$ to $\mathrm{BS})$, while considering uplink propagation.

5. In order to avoid energy exhaustion of cluster heads, a reselection is necessary.

There are some components using in our design.

1. Cluster head devices $(\mathrm{CHs}): \mathrm{CHs}$ are the leaders of a cluster, they collect the data from the group member devices and transmit aggregated data to the base station.

2. Group member devices (GMs): GMs are assigned to corresponding clusters formations and transmit the data to their respective $\mathrm{CH}$.

3. Intra communication: The data propagation between the group members and their respective cluster head.

4. Inter communication: The data propagation between cluster head and base station.

\subsection{Methodology}

Our research methodology requires developing a mathematical model for the whole system energy consumption and then finding the optimal number of cluster heads by using a mathematical derivation. Meanwhile, a system simulation model is set up in Matlab 
and numerical results to evaluate the performance are obtained by simulations. Finally, quantitative and qualitative analysis are given. 


\section{Chapter 3}

\section{System Model}

For energy- efficient communications, it is desirable to maximize the amount of data sent with a given amount of energy. In this chapter, we formulate the problem of energyefficient link adaptation and obtain the optimal number of cluster heads to make system energy efficiency $U$ maximum. For simulating $n$ M2M devices, if $p$ is the probability for devices becoming cluster heads, $n p$ will be the number of cluster heads. Then the problem is changed to find optimal $p$ to make $U$ maximum.

When we set up M2M communications network, particularly in the initial stage of building network, sometimes we only know how many devices will be deployed in the cell and have not any information for devices position. In that case, the appropriate number of cluster heads is calculated by using expectation. Therefor general mathematical model design which can represent common situation is necessary.

Some basic formulas and knowledge from digital communications will be utilized to derive our mathematical model. Meanwhile, we also use several ideas related to optimization for calculating the optimal value of cluster heads number. Before we set up the mathematical model, some reasonable assumptions are given.

\subsection{Assumption}

In order to make the model reasonable and logic while guaranteeing the model accuracy, we make the following assumptions. 
1. The devices are homogeneously distributed within the cell, according to a spatial Poisson process of intensity $\lambda$ in a 2-dimensional space.

2. All devices transmit data within the same radio range $r_{d}$. In this radio range, we assume that the devices will transmit data by using short distance propagation. On the other hand, the ones out of this range will transmit data by using a long distance propagation model.

3. When group members select their respective cluster heads, they only consider the cluster head which is the most close to them.

4. The communication from each device follows a isotropic propagation model.

5. The base station is located at the center of the terrain.

6. $R_{c}$ is the radius of the base station.

7. There are two types of cluster head, one is normal cluster head device which has group members in its cluster group, another has not group members. All cluster heads are directly connected to the BS and group member only transmit data to its cluster head. There are no extra hops in intra-communication and intercommunication.

8. We assume the bandwidth of each cluster is same and the system bandwidth is evenly allocated to each cluster head.

\subsection{Building Energy Efficiency Mathematical Model}

Shown in Figure 3.1, the red circles represent the group member devices and the triangles represent the cluster head devices. After considering corresponding path loss and shadowing effect, the reach power for GM is $P(G M)$ and the reach power for $\mathrm{CH}$ is $P(C H)$.

Depending on the Shannon capacity, the reach power of group member and cluster head determine the data rate $R(G M)$ and $R(C H)$ respectively. As mention above, We want to find out how much value of $p$ (the percentage of M2M devices becoming cluster heads) 


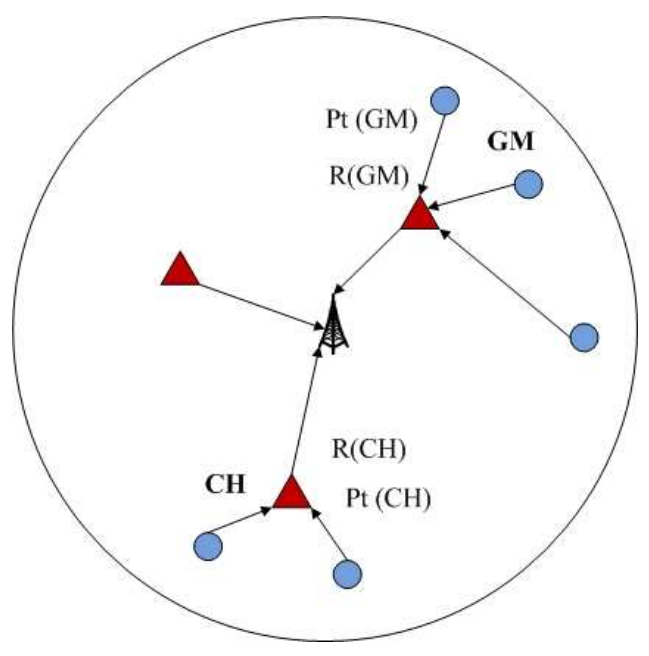

FiguRE 3.1: Transmit power for devices.

makes the whole system energy efficiency $U$ maximum:

$$
U(p)=\frac{R_{\text {system }}(p)}{P_{\text {system }}(p)}
$$

Where $R_{\text {system }}$ is the whole system data rate and $P_{\text {system }}$ is whole system power consumption. This function is respect to $p$.

\subsection{Mathematical Derivation}

In order to facilitate the calculation, we assume intra communication utilize short distance propagation model $P L(a)$ and corresponding shadow effect value $Z_{a}$. Meanwhile, for inter communication, using long distance propagation model $P L(e)$ and corresponding shadow effect value $Z_{e} . P L$ and $Z$ are $d B$ form which is defined in [22] and can be calculated as following.

$$
\begin{gathered}
P L(a)=28.5+20 \log \left(D_{2}\right) \\
P L(e)=128.1+37.6 \log \left(\frac{D_{1}}{1000}\right)
\end{gathered}
$$

Where $D_{1}$ is the distance in $m$ between cluster head and base station and $D_{2}$ is the distance in $m$ between group member and cluster head. 
In here, we provide a reasonable value for $P_{t}(G M)$, simultaneously, considering cluster head gathering data from group member and transmitting them in a long distance, we let $P_{t}(C H)$ is $\beta$ times $P_{t}(G M)$, moreover $\beta$ is large than 1 . Then we obtain the reach power for each device

$$
P_{J}(G M)=P_{t}(G M) \times 10^{-\frac{P L(a)_{J}+Z_{a}}{10}}
$$

$$
P_{K}(C H)=\beta P_{t}(G M) \times 10^{-\frac{P L(e)_{K}+Z_{e}}{10}}
$$

Where $J$ indicates the $J^{\text {th }}$ group member and $K$ indicates the $K^{\text {th }}$ cluster head.

By using equation (3.4), (3.5) and Shannon theory, the data rate for each cluster head and group member can be written as:

$$
\begin{aligned}
R_{J}(G M) & =\frac{w_{2}}{M_{K}+1} \log \left(1+\frac{P_{J}(G M)}{N_{0} \frac{w_{2}}{M_{K}+1}}\right) \\
= & \frac{w_{2}}{M_{K}+1} \log \left(1+\frac{P_{t}(G M)\left(M_{K}+1\right)}{N_{0} w_{2} 10 \frac{P L(a)_{J}+Z_{a}}{10}}\right) \\
R_{K}(C H) & =\frac{w_{1}}{n p} \log \left(1+\frac{P_{K}(C H)}{N_{0} \frac{w_{1}}{n p}}\right) \\
& =\frac{w_{1}}{n p} \log \left(1+\frac{\beta P_{t}(G M) n p}{N_{0} w_{1} 10 \frac{P L(e)_{K}+Z_{e}}{10}}\right)
\end{aligned}
$$

Where $w_{2}$ is the bandwidth of each cluster, we assume the bandwidth of each cluster is same. $w_{1}$ is bandwidth of whole system, we assume that system bandwidth is allocated evenly to each cluster head. $n$ is the number of M2M devices in the cell, $n p$ is the number of cluster head in the system. $M_{K}$ is the amount of group members for corresponding cluster head $K$ (not include cluster head itself). Therefor $\frac{w_{2}}{M_{K}+1}$ is the bandwidth which is allocated to each member in $K^{\text {th }}$ cluster. Moreover let the circuit power $P_{c}$ represents the average energy consumption of device electronics, the overall power consumption for group member and cluster head are

$$
P_{o}(G M)=P_{c}+P_{t}(G M)
$$




$$
P_{o}(C H)=P_{c}+P_{t}(C H)
$$

combining (3.8) and (3.9), and we have already know the number of cluster heads and group members in the whole system are $n p$ and $n(1-p)$ respectively. Therefor whole system power consumption is

$$
\begin{aligned}
P_{\text {system }}(p) & =p n P_{o}(C H)+(1-p) n P_{o}(G M) \\
& =n\left(P_{c}+P_{t}(G M)\right)+n P_{t}(G M)(\beta-1) p
\end{aligned}
$$

Meanwhile, the data rate for whole system is

$$
R_{\text {system }}(p)=\sum_{K=1}^{p n} R_{K}(C H)+\sum_{J=1}^{(1-p) n} R_{J}(G M)
$$

The Table 3.1 lists the parameters which will be utilized in our simulation.

\subsection{Expectation for Path loss and Cluster Size}

Based on (3.10) and (3.11), we realize the value of whole system power consumption depending on different $p$, but every device data rates are based on their locations and bandwidths. If we want to compute the system data rate by using (3.6) (3.7) and (3.11), we need to figure out every devices propagation path loss first, then obtain every devices bandwidths, which is equal to know the number of each cluster group members. So following, we try to derive a reasonable expected value for path loss of each device and cluster size of each group.

The M2M devices are distributed according to a homogeneous spatial Poisson process. The number of devices in a circle area with radius $R_{c}$ is a Poisson random variable, $N$, with mean $\lambda S$ where $S=\pi \times R_{c}^{2}$ and $\lambda$ is intensity of this Poisson process . Lets assume that for a particular realization of the process, there are $n$ devices in this area. As mention before, the probability of one device becoming a cluster head device $(\mathrm{CH})$ is $p$. Therefor, on average, there are $n p$ devices will become CHs. Also, the CHs and GMs are distributed as per independent homogeneous spatial Poisson processes $P 1$ and $P 0$ of intensity $\lambda_{1}=p \lambda$ and $\lambda_{0}=(1-p) \lambda[23]$. 
TABLE 3.1: System parameters [1-4]

\begin{tabular}{|c|c|}
\hline Shadowing (inter),$Z_{e}$ & $6 \mathrm{~dB}$ \\
Shadowing (intra), $Z_{a}$ & $3 \mathrm{~dB}$ \\
Thermal noise power, $N_{0}$ & $-204 \mathrm{dBW} / \mathrm{Hz}$ \\
Path loss (Long distance),$P L(e)$ & $128.1+37.6 \log \left(\frac{D_{1}}{1000}\right) \mathrm{dB}, D_{1}$ in m \\
Path loss (Short distance), $P L(a)$ & $38.5+20 \log D_{2} \mathrm{~dB}, D_{2}$ in $\mathrm{m}$ \\
System bandwidth, $w_{1}$ & $20 \mathrm{MHz}$ \\
Cluster bandwidth, $w_{2}$ & $200 \mathrm{KHz}$ \\
Number of devices, $n$ & 6000 \\
M2M Application & Smart meter \\
How often send packet, $t_{0}$ & $9090 \mathrm{~second}$ \\
Pack size, $l$ & $16136 \mathrm{bit}$ \\
Wake power, $P_{w}$ & $13.5 \mathrm{~mW}$ \\
Sleep power, $P_{s}$ & $15 \mu \mathrm{W}$ \\
Cell radius, $R_{c}$ & $800 \mathrm{~m}$ \\
Device radius, $r_{d}$ & $10 \mathrm{~m}$ \\
GM transmit power, $P_{t}(G M)$ & $0.01 \mathrm{~W}$ \\
Circuit power, $P_{c}$ & $-25 \mathrm{~dB}$ \\
Power ratio, $\beta$ & 30 \\
Cost parameter, $\omega$ & 5 \\
Aggregation parameter, $\alpha$ & 0.5 \\
Battery, $E_{b a t t e r y}$ & $500 \mathrm{~J}$ \\
\hline
\end{tabular}

Using the ideas from Voronoi tessellation [23], each device joins the cluster of the closest $\mathrm{CH}$ to form a Voronoi tessellation. The plane divides into zones called Voronoi cells, with each cell corresponding to a $P 1$ process point termed its nucleus. If $M$ is the random variable representing the number of $P 0$ process points in each Voronoi cell and $L_{v}$ is the total length of all segments connecting the $P_{0}$ process points to the nucleus in a Voronoi cell, then based on the results in [18]:

$$
\begin{gathered}
E[M \mid N=n] \approx \frac{1-p}{p} \\
E\left[L_{V} \mid N=n\right] \approx \frac{1-p}{2 p^{\frac{3}{2}} \sqrt{\lambda}}
\end{gathered}
$$


In here $E[M \mid N=n]$ is equal to the average value of cluster size. Therefor the average value of $D_{2}$ which is the distance between cluster head and it's group member is

$$
\begin{aligned}
E\left[D_{2} \mid N=n\right] & =\frac{E\left[L_{V} \mid N=n\right]}{E[M \mid N=n]} \\
& =\frac{\frac{1-p}{2 p^{\frac{3}{2}} \sqrt{\lambda}}}{\frac{1-p}{p}} \\
& =\frac{1}{2 \sqrt{\lambda p}}
\end{aligned}
$$

Let $D_{1}$ be a random variable that denotes the length of the segment from a device located at $(x, y)$ to the BS. a device could be located at any $(x, y)$ point on the terrain with uniform intensity, the probability density function of its location is constant $\frac{1}{S}$. So the average distance to the BS is:

$$
E\left[D_{1} \mid N=n\right]=\int_{s} \sqrt{x^{2}+y^{2}} \frac{1}{\pi R_{c}^{2}} d s=\frac{2}{3} R_{c}
$$

Now, by using (3.2) (3.3) (3.14) and (3.15), the expectation for $P L(e)$ and $P L(a)$ will be

$$
\begin{aligned}
E[P L(e) \mid N=n] & =128.1+37.6 \log \left(\frac{E\left[D_{1} \mid N=n\right]}{1000}\right) \\
& =128.1+37.6 \log \left(\frac{2 R_{c}}{3000}\right) \\
E[P L(a) \mid N n] & =38.5+20 \log E\left[D_{2} \mid N=n\right] \\
& =38.5+20 \log \left(\frac{1}{2 \sqrt{\lambda p}}\right)
\end{aligned}
$$

So far, we can derive the expectation for each cluster head data rate by using (3.7) and (3.16).

$$
E[R(C H) \mid N=n]=\frac{w_{1}}{n p} \log \left(1+\frac{\beta P_{t}(G M) n p}{N_{0} w_{1} 10^{\frac{E[P L(e) \mid N=n]+Z_{e}}{10}}}\right)
$$


Meanwhile the average data rate of each group member is

$$
E[R(G M) \mid N=n]=\frac{w_{2}}{E[M \mid N=n]+1} \log \left(1+\frac{P_{t}(G M)(E[M \mid N=n]+1)}{N_{0} w_{2} 10^{\frac{E[P L(a) \mid N=n]+Z_{a}}{10}}}\right)
$$

\subsection{Optimal Value Derivation}

Combining the equations (3.10) (3.11) (3.18) and (3.19), the expectation for energy efficiency function can be derived like

$$
\begin{aligned}
E[U(p) \mid N=n]= & \frac{E\left[R_{\text {system }}(p) \mid N=n\right]}{P_{\text {system }}(p)} \\
= & \frac{n p E[R(C H) \mid N=n]+n(1-p) E[R(G M) \mid N=n]}{P_{\text {system }}(p)} \\
= & \frac{1}{P_{\text {system }}(p)}\left(\frac { n p w _ { 1 } } { n p } \operatorname { l o g } \left(1+\frac{P_{t}(G M) n \beta p}{\left.N_{0} w_{1} 10^{\frac{E[P L(e) \mid N=n]+Z_{e}}{10}}\right)}\right.\right. \\
& +\frac{w_{2} n(1-p)}{E[M \mid N=n]+1} \log \left(1+\frac{P_{t}(G M)(E[M \mid N=n]+1)}{\left.\left.N_{0} w_{2} 10^{\frac{E[P L(a) \mid N=n]+Z_{a}}{10}}\right)\right)}\right. \\
= & \frac{1}{P_{\text {system }}(p)}\left(w_{1} \log \left(1+\frac{P_{t}(G M) n \beta p}{\left.29.3695 \times N_{0} w_{1} R_{c}^{3.76}\right)}\right)\right. \\
& \left.+w_{2} n\left(p-p^{2}\right) \log \left(1+\frac{P_{t}(G M) \lambda}{3531.4 \times N_{0} w_{2}}\right)\right) \\
= & \frac{w_{1} \log \left(1+\frac{P_{t}(G M) n \beta p}{\left.29.365 \times N_{0} w_{1} R_{c}^{3.76}\right)}\right.}{n\left(P_{c}+P_{t}(G M)\right)+n P_{t}(G M)(\beta-1) p}+ \\
& \frac{w_{2} n\left(p-p^{2}\right) \log \left(1+\frac{P_{t}(G M) \lambda}{3531.4 \times N_{0} w_{2}}\right)}{n\left(P_{c}+P_{t}(G M)\right)+n P_{t}(G M)(\beta-1) p}
\end{aligned}
$$


Removing the conditioning on $N$ and we know $E[n]=\lambda \pi R_{c}^{2}$. Therefore, the energy efficiency of M2M system can be computed by:

$$
\begin{aligned}
E[U(p)]= & E[E[U(p) \mid N=n]] \\
= & \frac{w_{1} \log \left(1+\frac{P_{t}(G M) E[n] \beta p}{29.3695 \times N_{0} w_{1} R_{c}^{3.76}}\right)}{E[n]\left(P_{c}+P_{t}(G M)\right)+E[n] P_{t}(G M)(\beta-1) p}+ \\
& \frac{w_{2} E[n]\left(p-p^{2}\right) \log \left(1+\frac{P_{t}(G M) \lambda}{3531.4 \times N_{0} w_{2}}\right)}{E[n]\left(P_{c}+P_{t}(G M)\right)+E[n] P_{t}(G M)(\beta-1) p} \\
= & \frac{w_{1} \log \left(1+\frac{P_{t}(G M) \lambda \pi R_{c}^{2} \beta p}{\left.29.3695 \times N_{0} w_{1} R_{c}^{3.76}\right)}\right.}{\lambda \pi R_{c}^{2}\left(P_{c}+P_{t}(G M)\right)+\lambda \pi R_{c}^{2} P_{t}(G M)(\beta-1) p}+ \\
& \frac{w_{2} \lambda \pi R_{c}^{2}\left(p-p^{2}\right) \log \left(1+\frac{P_{t}(G M) \lambda}{3531.4 \times N_{0} w_{2}}\right)}{\lambda \pi R_{c}^{2}\left(P_{c}+P_{t}(G M)\right)+\lambda \pi R_{c}^{2} P_{t}(G M)(\beta-1) p} \\
= & \frac{w_{1} \log (1+A p)+B\left(p-p^{2}\right)}{C+D p}
\end{aligned}
$$

Where we denotes $A=\frac{\beta P_{t}(G M) \lambda \pi R_{c}^{2}}{29.3695 \times N_{0} w_{1} R_{c}^{3.76}}, B=\lambda \pi R_{c}^{2} w_{2} \log \left(1+\frac{P_{t}(G M) \lambda}{3531.4 \times N_{0} w_{2}}\right), C=$ $\lambda \pi R_{c}^{2}\left(P_{\text {circuit }}+P_{t}(G M)\right)$ and $D=\lambda \pi R_{c}^{2} P_{t}(G M)(\beta-1)$.

We let $E\left[U^{\prime}(p)\right]=0$ and obtain the optimal value of $p^{*}$ and corresponding $E\left[U\left(p^{*}\right)\right]$. Figure (3.2) demonstrates the relationship between the optimal $p$ and energy efficiency as different $\beta$. We can know $E[U(p)]$ is a continuous function over $[0,1]$, thus for a fixed number of $\beta$, max $E[U(p)]$ can only be taken at point within $\{0,1\} \bigcup\left\{p: E\left[U^{\prime}(p)\right]=0\right\}$. By using Matlab, it is easy to find this unique optimal $p^{*}$ corresponding to fixed value $\beta$.

Obtaining the expected optimal value $p^{*}$ is equal to find the approximate optimal number of cluster heads $\left(n p^{*}\right)$. There are two situations, when handling the intra communication. For static design, we utilize (3.21) to calculate optimal $p$. In here we assume all intra communication utilize short distance propagation model $P L(a)$ and corresponding shadow effect value $Z_{a}$. But for dynamic design, which kind of propagation model is used based on whether distance between devices are large than rd. Next chapter, we will utilized the expected optimal value $p^{*}$ to set up cluster, we name this way as static cluster design. Moreover we determine which devices will be cluster head by using cost function. 


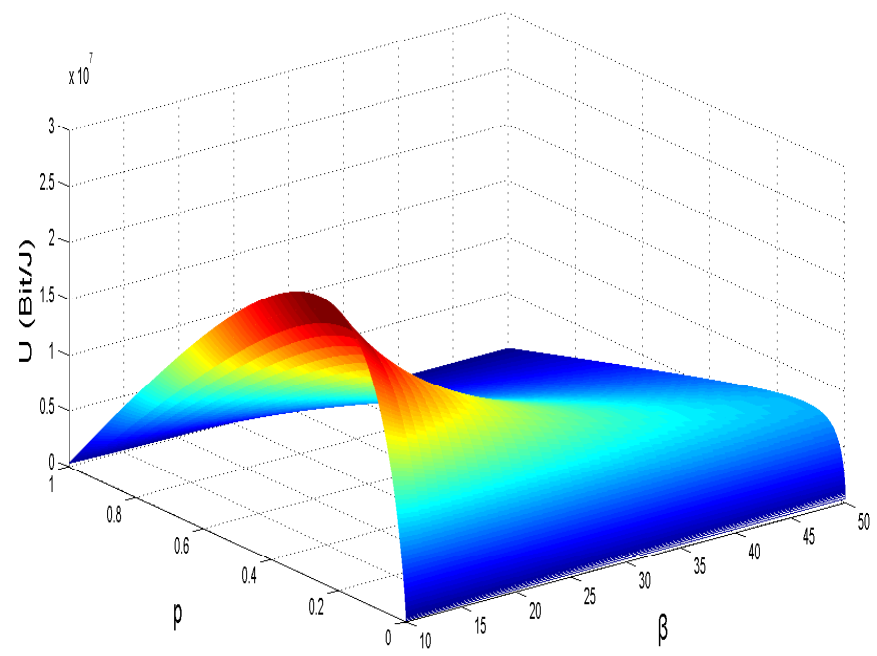

FiguRE 3.2: Energy efficiency with different $p$ and $\beta$. 


\section{Chapter 4}

\section{Static Cluster Design}

One key point for static cluster design is that we will not change $p$ value in the whole processing after obtaining the approximate optimal $p$. In this chapter, The basic procedure for building cluster are described. Referring to flow chart Figure (4.1), there are six main steps in the simulation. At the begin, the random devices are deployed in a cell. Then we utilize (3.21) to calculate optimal $p *$. In third and fourth step, depending on the number of cluster heads, the cell is divided into almost even squares and cluster heads are decided by using cost function. In order to make energy consumption for each device balance, reselecting cluster heads will be used in fifth step. At the next chapter, we will compare with the results by using different value $p$ cluster designs.

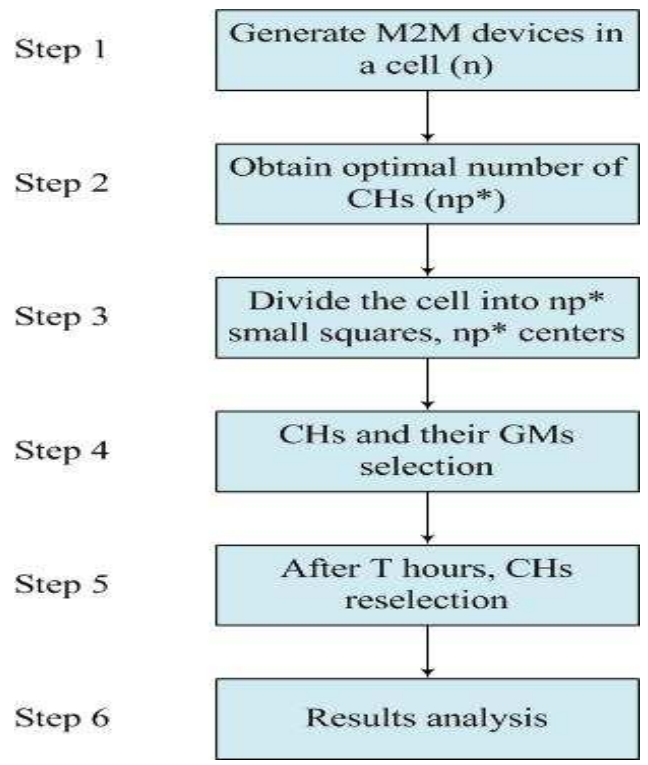

Figure 4.1: The flow chart for static cluster design. 


\subsection{Deploy Devices}

A reasonable number of devices are generated within the cell which radius is $R_{c}$. In here, the locations and initial energy capacities of every devices are initialized. Moreover, in order to identity every devices clearly, overlapping devices are avoided. Other parameters are also set up such as bandwidth, pass loss, shadowing effect and so on.

\subsection{The Number of Cluster Heads}

First, we add the parameters which are listed in Table (3.1) into (3.21). Therefor, we derive an energy efficiency function which has only two variables ( $p$ and $\beta)$. By simulating (3.21) in Matlab, we draw the Figure (3.2) that presents the relationship among $p, \beta$ and the value of $\mathrm{EE}$. The figure shows for each $\beta$, equation (3.21) is convex in $[0,1]$. It means there are only one maximum of $E[U]$ regarding to each value $\beta$, moreover it is not difficult to find corresponding $p^{*}$ by utilizing Matlab. Another point demonstrated by Figure (3.2) is bigger value of $\beta$ will lead to smaller value of energy efficiency $(U)$. Bigger $\beta$ means more power on each cluster head, different value of $\beta$ will change the parameter $A$ and $D$ in (3.21). Bigger $\beta$ leads to bigger $A$ which decides to larger data rate for the cluster head. Meanwhile, bigger $\beta$ also increases the parameter $D$ which indicates more power consumption for whole system. Comparing with more power consumption for cluster heads, the data rate of cluster heads does not increase significantly, which indicates energy efficiency will decrease.

Figure (4.2) demonstrates that different value $\beta$ leads to different value of optimal $p$. Increasing the value of $\beta$, we will obtain smaller optimal $p$. The reason is not complicated. When the system reaches the optimal state for energy efficiency, increasing the power of cluster heads (increase $\beta$ ) is not an efficient way for improving the whole system data rate. Therefor less number of cluster heads (decrease $p$ ) will make the whole power for cluster heads decrease and let whole system return to optimal state. 


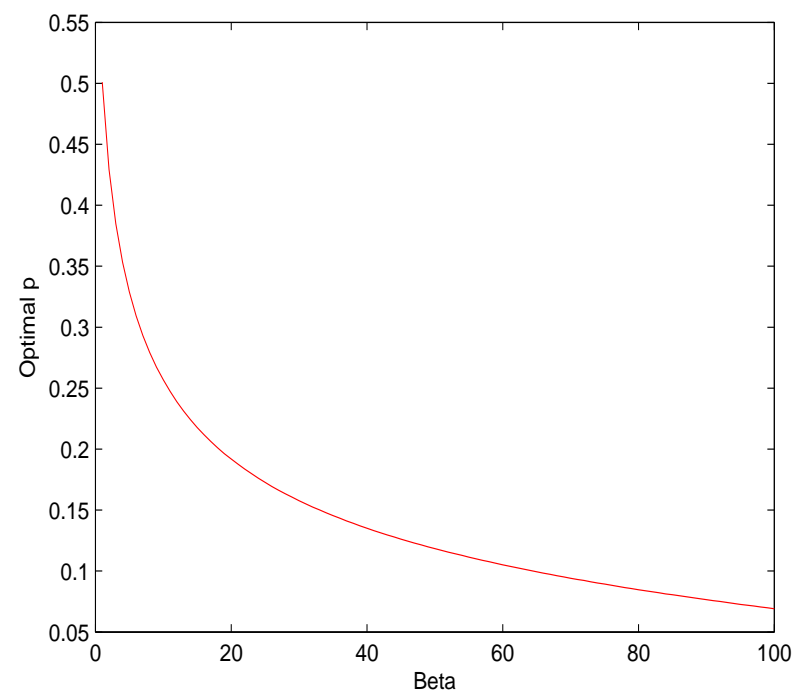

FiguRE 4.2: The relation between optimal $p$ and $\beta$ in static design.

\subsection{Cell Segmentation}

Since we have already figured out the value of $p^{*}$, the best choice for the number of cluster heads $\left(n p^{*}\right)$ is obtained. Like mentioned in chapter 2 , we want to guarantee the CHs are distributed as well as possible, therefor we will divide the cell into small squares as even as possible.

In the part of assumption, we assume the base station is located at the center of the cell. First at all, based on the four quadrants we divide the cell into four sectors, which are first, second, third and fourth quadrant. Therefor the total number of cluster heads $(n p *)$ are also divided into four parts. The first three quadrants have the same number of cluster heads which is $\left\lceil\frac{n p^{*}}{4}\right\rceil$, and the fourth quadrant has $n p^{*}-3\left\lceil\frac{n p^{*}}{4}\right\rceil$ cluster heads. Each quadrant is separated into small squares according to the allocated CHs amount. The area of small square in each quadrant is equal to the area of sector divided by corresponding $\mathrm{CHs}$ amount, moreover the length of square can also be calculated. For example in the first quadrant, the area of square is $\frac{\pi R_{c}^{2}}{4}$ divided by $\left\lceil\frac{n p^{*}}{4}\right\rceil$. Algorithm (1) is utilized to find every small square centers in the first quadrant.

Similar with Algorithm (1), we will figure out the square centers in other quadrants, and obtain a $n p^{*} \times 2$ matrix which contains the locations for every centers. After contrasting this matrix with every M2M devices position, we will know which devices are close to 


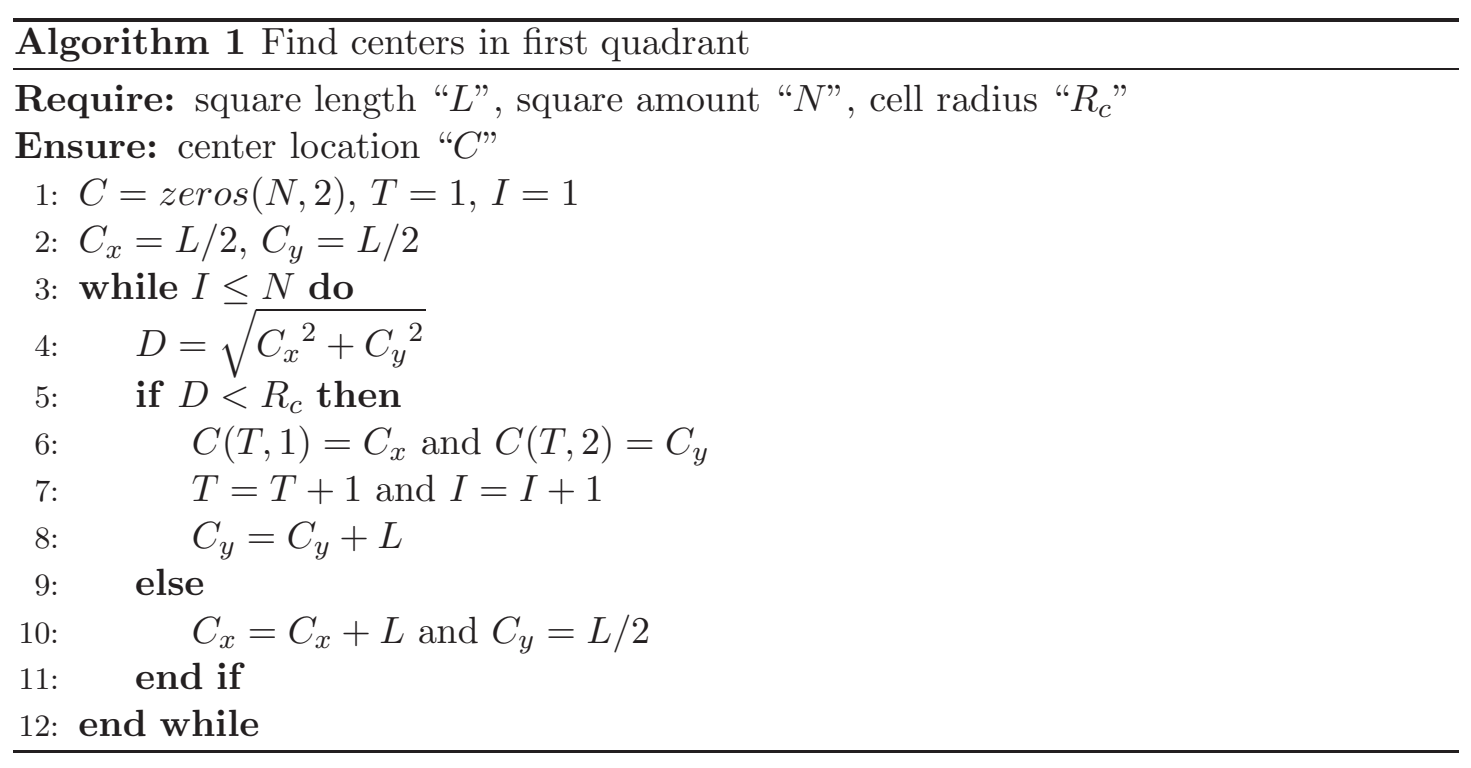

these centers individually. Shown in Figure (4.3), The red triangles are centers of every squares, the blue devices which close to centers are named reference devices (the same amount with cluster heads). Other normal M2M devices (black points) will choose the most close reference devices as their cluster groups. In each cluster group, every devices (include reference devices and normal devices) are potential cluster head. Which one will be really cluster head, based on our cost function that is introduced as following

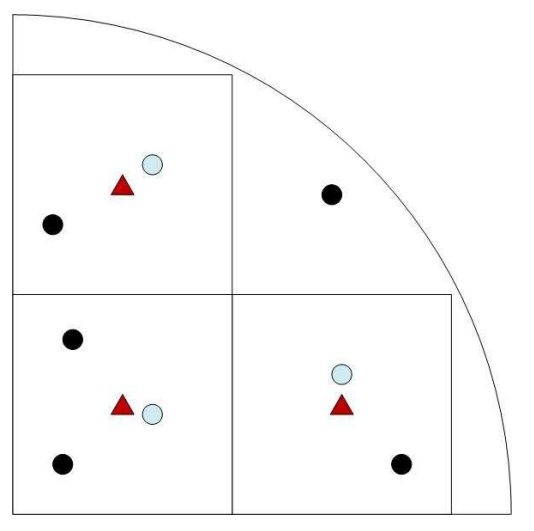

Figure 4.3: Segmentation in first quadrant.

\subsection{Cluster Head Selection}

Last section, we have already divided the M2M devices into $n p^{*}$ groups. In here, we will derive a cost function to select cluster heads in each group. At initial time, the function is considered in two factors that are intra communications and inter communications, 
moreover only one cluster head is determined in each group. Furthermore the cost function will be developed in the cluster head reselection part, because we also need to consider residual energy of each devices beside communication consumption.

\subsubsection{Inter Communication Cost}

As mentioned before, inter communication represents the propagation between cluster head and base station. Since we have already known every devices locations in each cluster group, it is easy to figure out every members long distance propagation consumptions by using (3.3). We assume every cluster heads have the same transmit power $P_{t}(\mathrm{CH})$, referring to (3.5), the reach power for each device only depends on the different path loss. Generally we believe cluster heads using long distance propagation model to transmit data, therefor inter cost $F_{i}$ is

$$
\begin{aligned}
F_{i} & =\frac{\left(M_{K}+1\right) P L(e)_{i}}{\sum_{j=1}^{M_{K}+1} P L(e)_{j}} \\
& =\frac{\left(M_{K}+1\right) D_{i}^{3.76}}{\sum_{j=1}^{M_{K}+1} D_{j}^{3.76}}
\end{aligned}
$$

$i$ is the $i^{\text {th }}$ group member in the cluster. Which has small value $F_{i}$ will consumes small energy in inter communication.

\subsubsection{Intra Communication Cost}

Intra communication is defined as data exchange between cluster head and group member. When evaluating the intra communication cost, we assume every group members utilize short distance propagation, $P L(a)$, to transmit information. In each cluster group, we assess intra consumption by regarding every devices as cluster head and finding which one has minimum intra cost.

For example, referring to Figure (4.4), if point 1 is selected as cluster head, first we calculate it's intra consumption which is $C(a)_{1}=P L(a)_{12}+P L(a)_{13}+P L(a)_{14}$. Similarly, we can obtain $C(a)_{2}, C(a)_{3}, C(a)_{4}$ respectively. Therefor the intra communication cost for point 1 in this group is $f_{1}=\frac{4 C(a)_{1}}{C(a)_{1}+C(a)_{2}+C(a)_{3}+C(a)_{4}}$. considering the general case, 


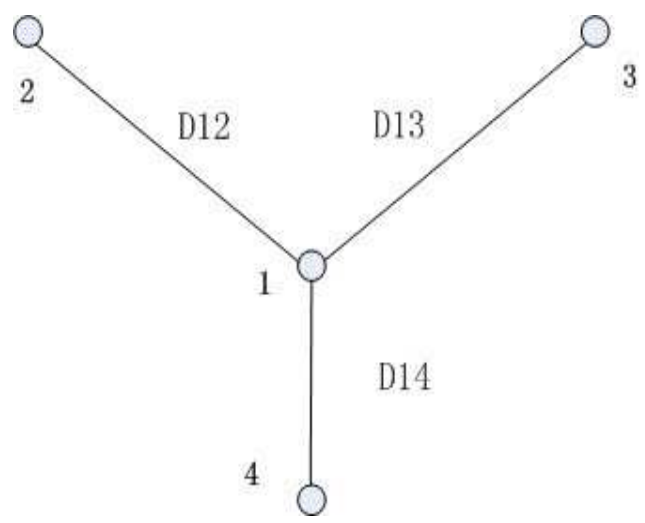

FiguRE 4.4: Intra consumption.

we derive following equation:

$$
\begin{aligned}
f_{i} & =\frac{\left(M_{K}+1\right) C(a)_{i}}{\sum_{j=1}^{M_{K}+1} C(a)_{j}} \\
& =\frac{\left(M_{K}+1\right) \sum_{j=1}^{M_{K}+1} D_{i j}^{2}}{\sum_{k=1}^{M_{K}+1} \sum_{j=1}^{M_{K}+1} D_{k j}^{2}}
\end{aligned}
$$

Where $D_{k j}$ is the distance between member $k$ and other members, $M_{K}$ is the number for group member. This function $f_{i}$ is considered for the aspect of short distance propagation.

\subsubsection{Cost Function}

Combining intra and inter communication, cost function is given by:

$$
\text { cost }_{i}=f_{i}+F_{i}
$$

The device which has the minimum cost means the device using the least communication consumption, therefor it will be selected as cluster head in the group at the initial state. The same thing happen for other cluster groups, each group selects one device which has minimum cost as it's cluster head. As a result, we can find which devices are cluster heads by using (4.3), meanwhile get their locations. Additionally, other normal devices will select the most close cluster head as their heads. Cluster are formed by using Algorithm (2). By using this algorithm, we obtain the cluster heads locations, their group members locations and cluster size. See Figure (4.5) as an example for part of cluster, in here red circles are cluster heads and blue points are group members. Based 
on the figure, we can see different cluster groups have different cluster size, moreover some cluster heads even have no members in their groups.

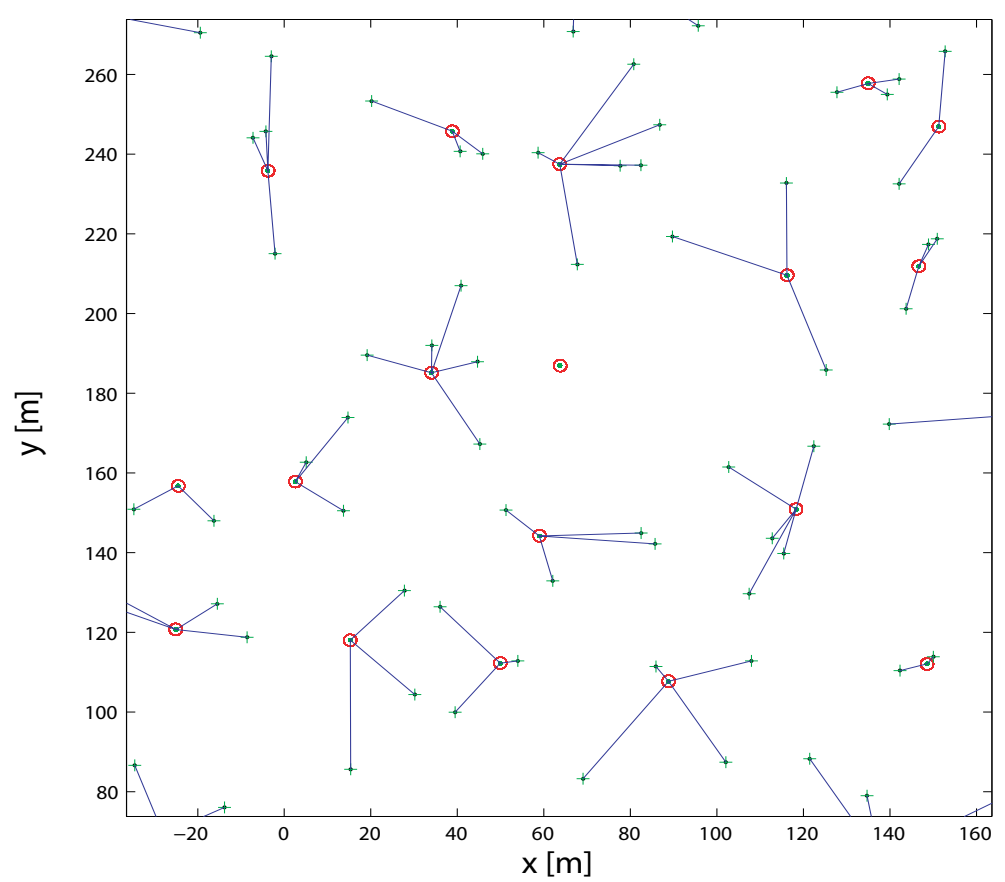

FiguRE 4.5: Cluster formation.

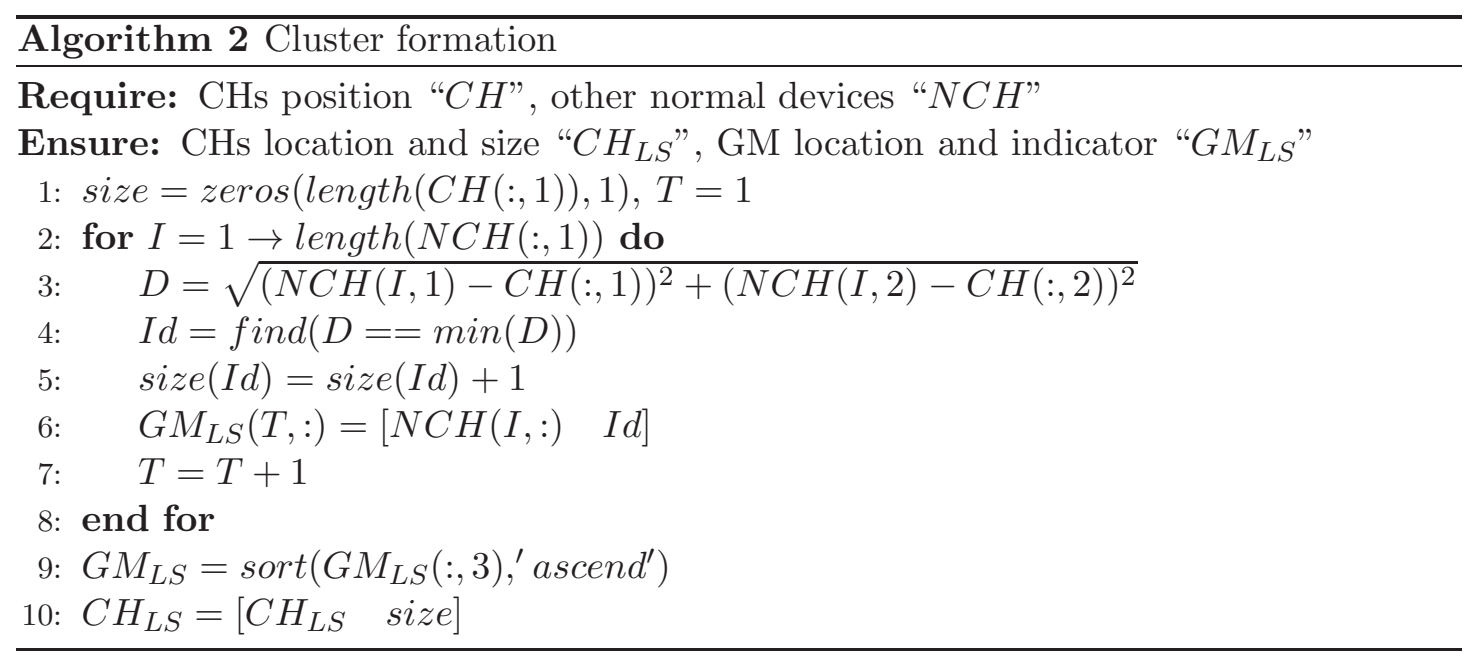

As we know, group members transmit their packets to their cluster heads, moreover cluster heads aggregate these packets and forward them to base station. At the initial time, the battery energy for every devices are same, therefor we do not consider residual energy for every device in this state. But in the reselection step, M2M devices will have different residual energy after $T$ hours. The reason is obviously, every devices have different propagation distance, furthermore cluster heads and group members utilized 
different transmit power. We will develop the cost function in the next part to handle this problem.

\subsection{Cluster Head Reselection}

In the above analysis, cluster head use much more power to transmit than group member and forward aggregation packets which normally contain more than one packets. If the device is cluster head at whole operation time, it's energy will be exhausted faster than normal devices, which does not meet our goal that every devices have similar life time. Therefor, cluster head reselection is an effective way to reach the requirement. Before developing cost function, a detailed description for device energy consumption is given.

\subsubsection{Energy Consumption for Device}

With regard to group members, We assume device will wake up when transmitting data and sleep in other time. The wake time is $\frac{l}{R_{J}(G M)}$, in here $l$ is packet size and $R_{J}(G M)$ is device's data rate. Therefor the energy consumption of the group member $J$ in $T$ hours is given by

$$
C_{J}(G M)=\frac{T}{t_{0}}\left(\frac{P_{o}(G M) l}{R_{J}(G M)}+\frac{P_{w} l}{R_{J}(G M)}+P_{s}\left(t_{0}-\frac{l}{R_{J}(G M)}\right)\right)
$$

Where $P_{w}$ is wake power consumption and $P_{s}$ is sleep power consumption, $t_{0}$ is the device's interval time between two events, it represents device sent packet every $t_{0}$ time. Similar for cluster head, the energy consumption of $K^{\text {th }} \mathrm{CH}$ is

$$
C_{K}(C H)=\frac{T}{t_{0}}\left(\frac{P_{o}(C H) \alpha\left(M_{K}+1\right) l}{R_{K}(C H)}+\frac{P_{w} \alpha\left(M_{K}+1\right) l}{R_{K}(C H)}+P_{s}\left(t_{0}-\frac{\alpha\left(M_{K}+1\right) l}{R_{K}(C H)}\right)\right)
$$

$M_{K}$ is group member amount for the $K^{\text {th }}$ cluster, $\alpha$ is aggregation parameter for cluster head. In [24], the writer assume a perfect aggregation which makes the cluster head compresses all receiving packets and itself into one packet and forward it to base station. But for really communication systems, a $1: N$ data aggregation is not often the appropriate model. It is important to know which kinds of M2M applications are simulated, different applications may result to different aggregation values. So in our case, 
we utilize parameter $\alpha$ to show possible aggregation value. Combining (4.4) and (4.5), we derive the whole system consumption after $T$ hours

$$
C(\text { system })=\sum_{K=1}^{n p}\left(C_{K}(C H)+\sum_{J=1}^{M_{K}} C_{K J}(G M)\right)
$$

\subsubsection{Cluster Head Reselection}

According to (4.4) and (4.5), it is easy to figure out every devices energy consumptions including transmit consumption, wake up consumption and sleep consumption. After $T$ time, the consumption of each devices $E_{i}$ can be obtained. Therefor, we normalize each device's consumption in it's cluster group and get $E_{i}$

$$
E_{i}=\frac{\left(M_{K}+1\right) E_{i}}{\sum_{j=1}^{M_{K}+1} E_{j}}
$$

Furthermore the cost function is developed by:

$$
C O S T_{i}=f_{i}+F_{i}+\omega E_{i}
$$

$\omega$ is used for making the third part of equation outstanding. As a result, in each cluster group, which devices has minimum COST will be cluster head in next round. 


\section{Chapter 5}

\section{Performance Analysis for Static Cluster Design}

In previous chapter, we describe how static cluster design works. As mentioned in Section 3.5, when utilizing (3.21) to obtain optimal value $p$, we assume every intra communications use short distance propagation, it means we assume every distances between cluster heads and group members are less than $r_{d}$. It is obviously that the optimal value of $p$ obtained by (3.21) is more accurate as increasing the intensity $\lambda$. We assume that the M2M network is deployed in a fixed area, therefor high intensity is same as large number of M2M devices. For this reason, we deployed 6000 devices in one cell. We compare the energy consumption results between static cluster design and Non-cluster design (devices connect BS directly), in order to show cluster design is an energy efficient way. Furthermore, we concentrate on discussing different cluster designs which use different $p$ values to prove that an appropriate number of cluster heads can save energy most. Performance of different designs are evaluated through graphical analysis and numerical discussion. The parameters in our simulation are based on table 3.1 .

\subsection{Residual Energy Analysis}

For static cluster design, we only calculate the optimal value of $p$ at initial time and will not change this value at following step, even some dead devices appear. After simulating 
(3.21) in Matlab, we know that the expected value of $U$ have maximum value when $p$ equal to 0.1577 . Therefor, for simulating 6000 devices, the appropriate number of cluster heads are 947 and the remain number of devices will be group members. Based on cluster head selection and cost function, we decide which devices are cluster heads, meanwhile the remain devices are assigned to each cluster group. It is easy to obtain the energy consumption for each device, moreover we calculate the whole system consumption by using (4.6). Figure (5.1) represents the consumption results for different $p$ in 30 days. In the figure, the red line represents the system residual energy when $p$ equals to 0.1577 .

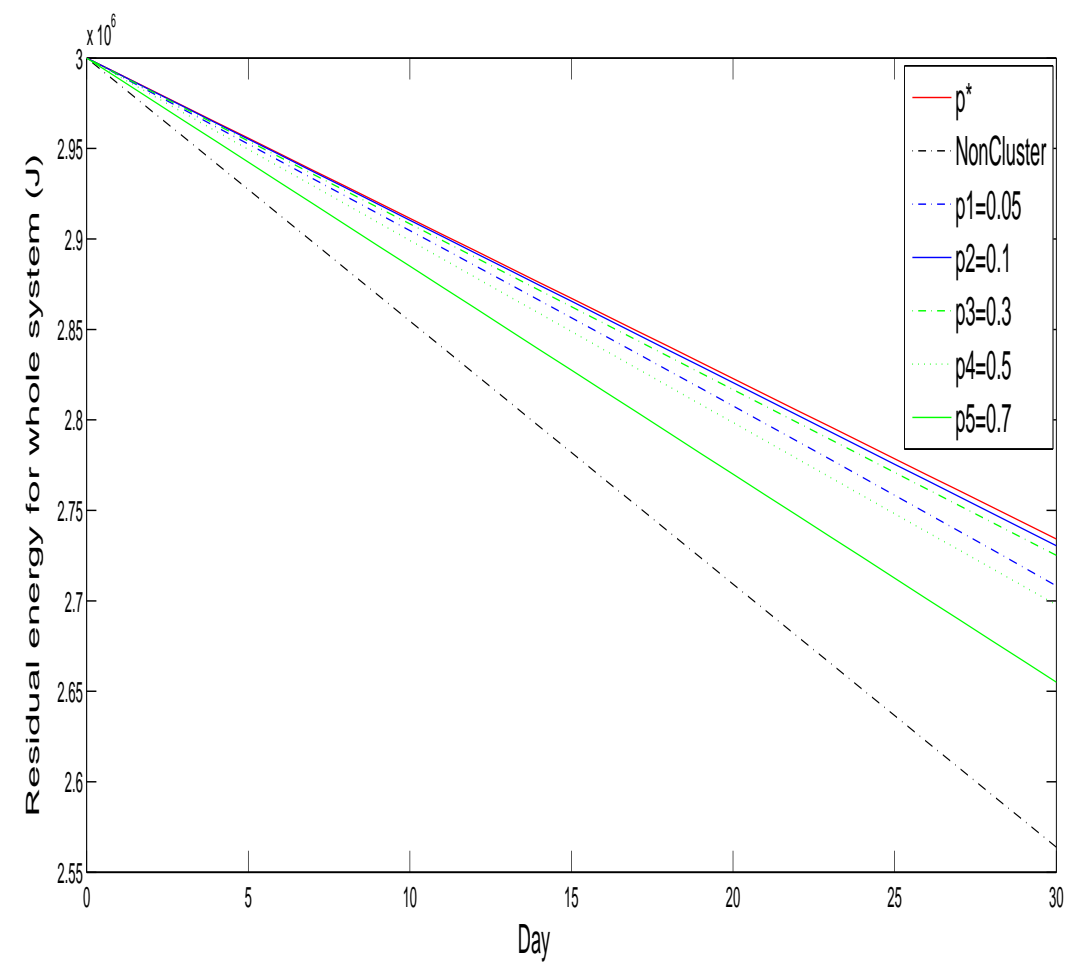

FiguRE 5.1: Whole system residual energy for different $p$ in 30 days.

During 30 days, it remains the highest value for residual energy comparing with other six designs. The blue solid line and the blue dot-dashed line represent $p=0.1$ and $p=0.05$ respectively, because $p=0.1$ is closer to optimal $p$ than $p=0.05$, it's residual energy is higher than another. The same result happens when $p$ larger than 0.1577 , the design which value $(p=0.3)$ is close to 0.1577 has good performance comparing with $p=0.5$ and $p=0.7$. Results of Figure (5.1) indicate that the optimal number of cluster heads is existence and the static design is an efficient way to find it. Moreover, Non-cluster design (NonCluster) consumes the highest energy comparing with other cluster designs. 
Figure (5.2) shows the whole system residual energy by using different $p$ designs on $100^{\text {th }}$, $200^{\text {th }}$ and $300^{\text {th }}$ day. The light blue bar (optimal $p$ ) has the optimal performance on the three periods, the remain energy is highest comparing with other values $p$. NonCluster design (Navy color) has the worst performance, this result shows that directly connecting base station is not a good way for energy saving. Since we know $p^{*}=0.1577, p_{2}=0.1$ and $p_{3}=0.3$ which are close to optimal $p$ have the second good performance, their residual energy are close to static design. Moreover, other $p$ value designs have worse result, since they are far away to $p^{*}$. This figure is an evidence that proves our mathematical model $(3.21)$ is convex one in $[0,1]$ and the value obtained by us is approximate optimal value.

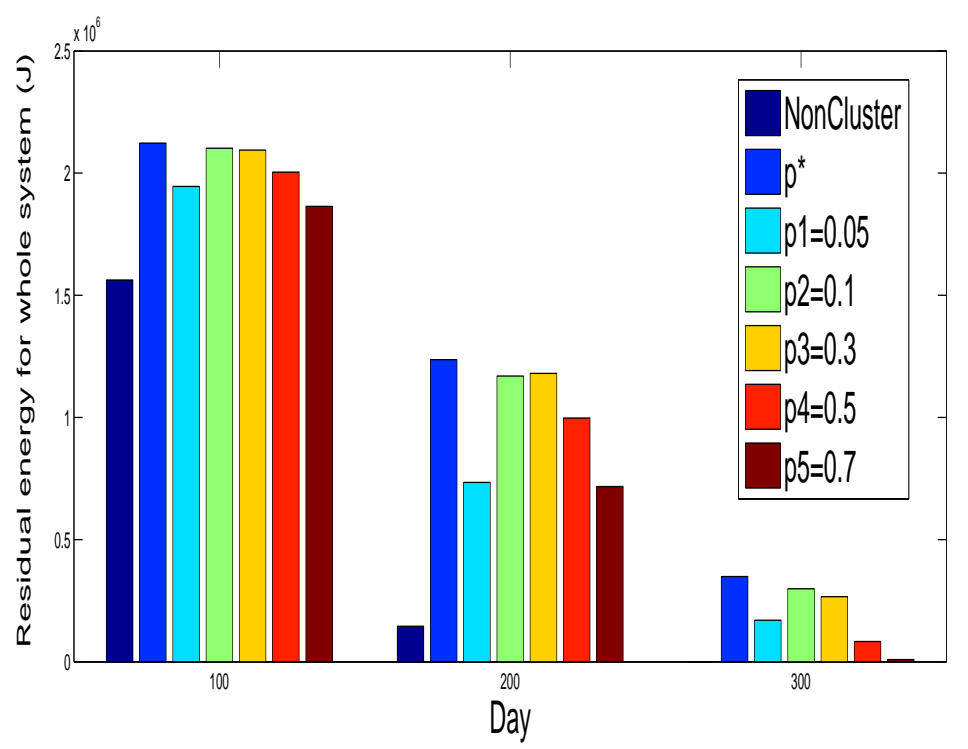

Figure 5.2: Residual energy for different $p$ on $100^{\text {th }}, 200^{\text {th }}, 300^{\text {th }}$ day.

Table (5.1) gives a numerical description for Figure (5.2) on three different periods. Based on table, we can see static design has a significant improvement for saving energy comparing with direct connection. Since we simulate 6000 devices and every devices have $500 \mathrm{~J}$ energy in initial time, therefor whole system energy at beginning is $3 \times 10^{6}$ J. On the $100^{\text {th }}$ day, NonCluster only remains $52 \%$ of whole energy, but static design has $71 \%$ of whole energy. The same situation on $300^{\text {th }}$ day, NonCluster almost runs out of energy, while static cluster design system still can be operated. Another interesting thing is NonCluster consume more energy at beginning than later period. The reason is at later period, the devices which far away to base station are dead, because of long distance propagation, therefor the whole system consumption become smaller. It means 
the distribution of energy consumption is not even. But for static design, Basically, it do not have this shortage.

TABLE 5.1: System residual energy on different days

\begin{tabular}{|c|c|c|c|}
\hline Design way & $100^{\text {th }}$ day & $200^{\text {th }}$ day & $300^{\text {th }}$ day \\
\hline$p^{*}$ & $2.12 \times 10^{6}$ & $1.23 \times 10^{6}$ & $3.43 \times 10^{5}$ \\
NonCluster & $1.56 \times 10^{6}$ & $1.49 \times 10^{5}$ & 52.15 \\
$p_{1}=0.05$ & $1.92 \times 10^{6}$ & $7.11 \times 10^{5}$ & $1.66 \times 10^{5}$ \\
$p_{2}=0.1$ & $2.10 \times 10^{6}$ & $1.16 \times 10^{6}$ & $2.93 \times 10^{5}$ \\
$p_{3}=0.3$ & $2.09 \times 10^{6}$ & $1.18 \times 10^{6}$ & $2.63 \times 10^{5}$ \\
$p_{4}=0.5$ & $2.0 \times 10^{6}$ & $9.96 \times 10^{5}$ & $8.11 \times 10^{4}$ \\
$p_{5}=0.7$ & $1.86 \times 10^{6}$ & $7.12 \times 10^{5}$ & $6.73 \times 10^{3}$ \\
\hline
\end{tabular}

\subsection{Dead Devices and Lifetime Analysis}

Like mentioned in chapter 1 , if we assume the recharging and replacing the battery are impossible, after a long running time, system will appear more and more dead devices. Based on our simulation, there are there ways to handle this problem depending on different situation.

1. If the dead device is group member, we will count the number of dead devices and remove it from system operation.

2. If the dead device is cluster head which has group members, we will count the number of dead devices and find a reasonable device from it's group member to replace it, then remove dead one from system operation.

3. If the dead device is cluster head which does not have group members, we will count the number of dead devices and remove dead one from system operation.

Algorithm 3 illustrates the step above. In here, $\mathrm{CH}$ is matrix which saves all cluster heads and $G M$ is the matrix which saves all group members. Both of them are 4 columns matrix, the first two columns are locations, the third one is used to save residual energy, the final columns of $C H$ is utilized for saving the size of corresponding cluster, the final columns of $G M$ is utilized for saving indicator which indicate the address of 


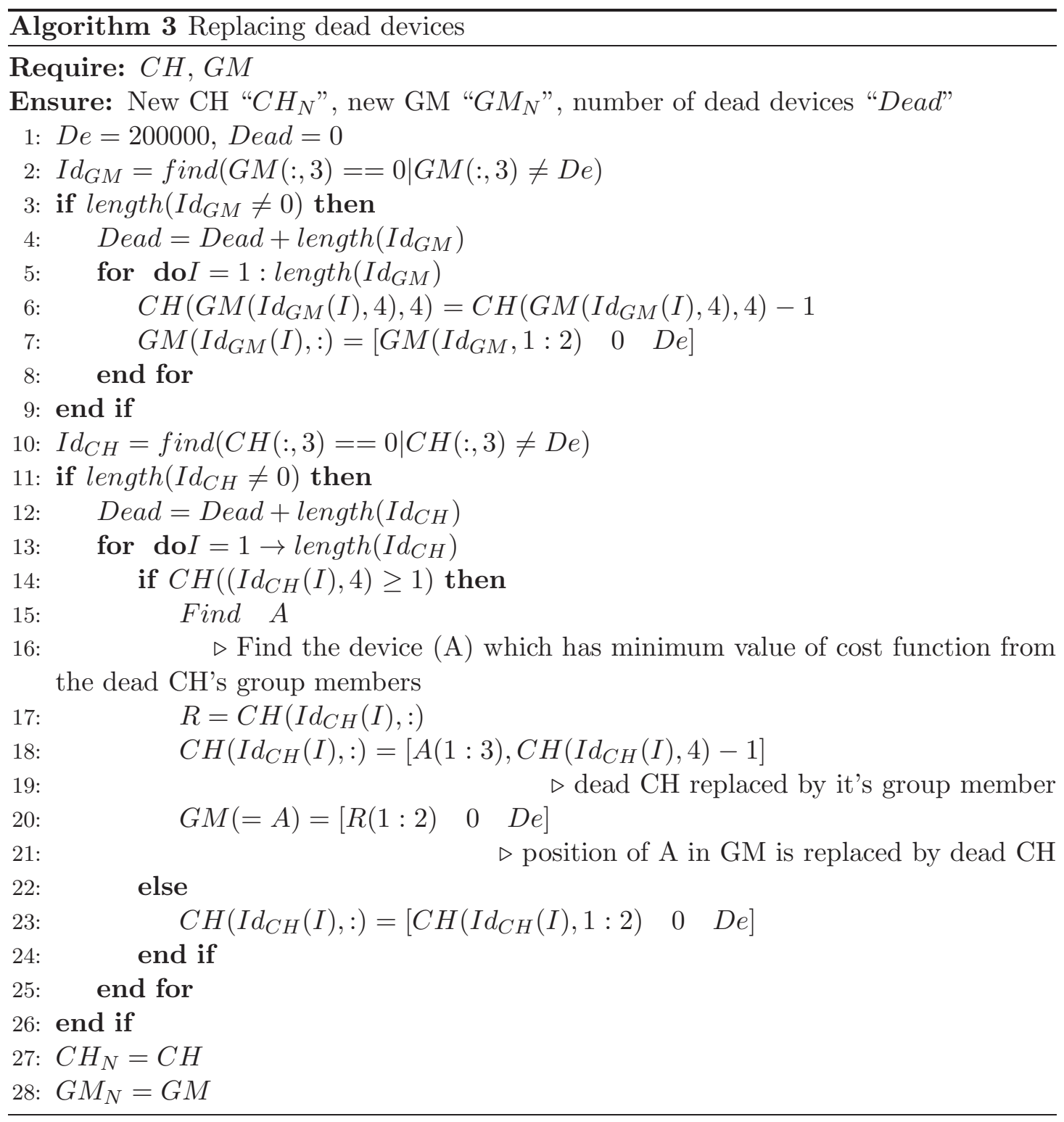

corresponding cluster head. De is sign of the dead device, every devices which do not have any energy will be marked De instead of residual energy in the third columns. When replacing the dead cluster head, we use $A$ to represent dead CH's group member which has minimum value of (4.8). Therefor, after using the Algorithm (3), we obtain the Figure (5.3) to compare dead devices results in different cluster designs.

Figure (5.3) shows two interesting performances. The first, $p=0.05$ design which is the blue solid line first begins to appear dead devices after system running about 150 days. The reason is not complicated, comparing with other cluster design, $p=0.05$ design has the least number of cluster heads, therefor cluster heads have much more group members than other designs. Every cluster heads which have a large number of group members will consume more energy to forward their packets, therefor some devices which become 


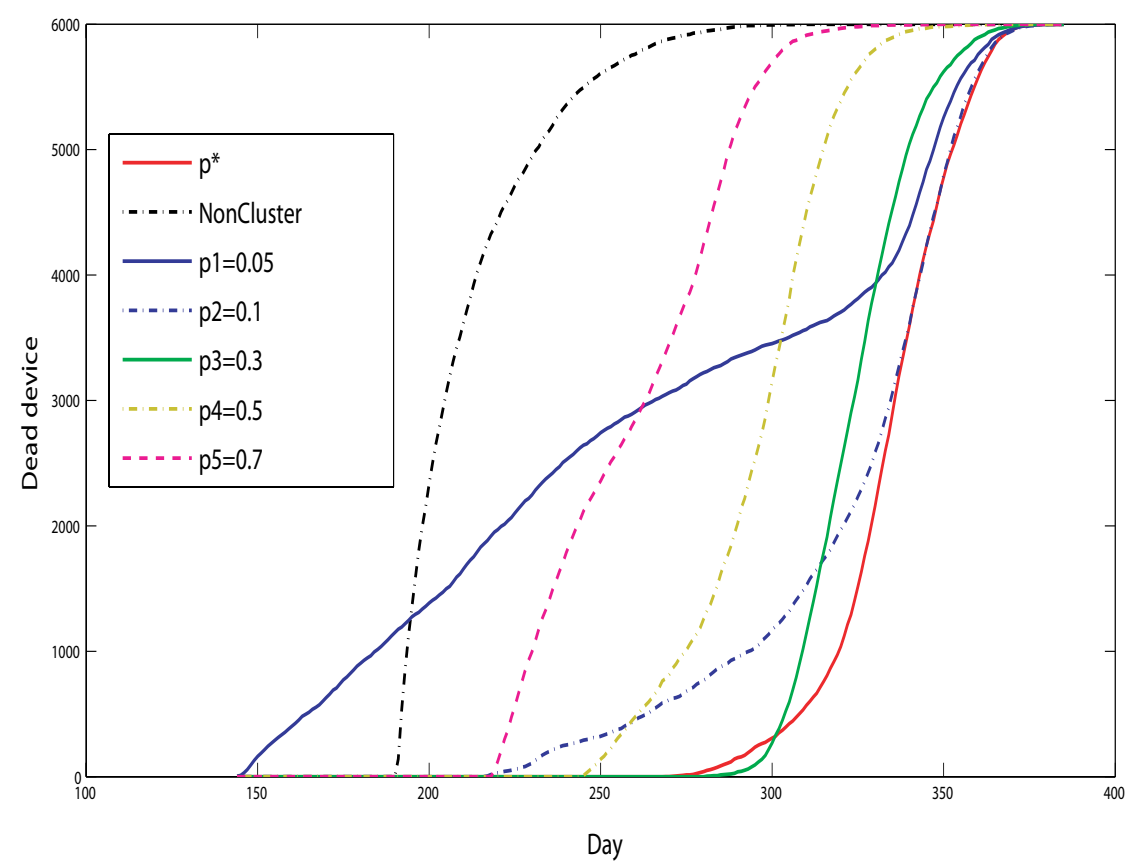

Figure 5.3: Dead devices with different $p$.

cluster heads more frequently, have large amount group members and far away to base station will be dead earlier.

The second, Non-Cluster design which is the black dotted line has the worst performance, most of it's devices are dead on around $250^{\text {th }}$ day. Static design has the best performance during all time. There is a special case, from $270^{\text {th }}$ to $300^{\text {th }}$ day, static design has a little more dead devices than $p=0.3$ design. Because comparing with the devices belong to $p=0.3$ design, the devices (in static design) which are far away to base station have more group member and will be dead earlier, but other devices save the energy, therefor after 300 days, since lots of devices energy in $p=0.3$ design are almost exhausted, more and more dead devices appear, the static design has better performance than $p=0.3$ design.

Results of our analysis indicate that the smallest value $p$ design or the biggest value $p$ design dose not mean the most saving energy design, it exists an appropriate value $p$ to make whole system consuming minimal energy.

As we mentioned before, the system network lifetime can be defined as most of 'dead' devices (such as $90 \%$ devices 'died'). We compare the system running time among different design and shown in Figure 5.4. Based on the figure, we can see static design has the best performance and Non-cluster has the least lifetime. The result shows that 
with simple static energy-efficient clustering operations, the network life can be extended by about $50 \%$.

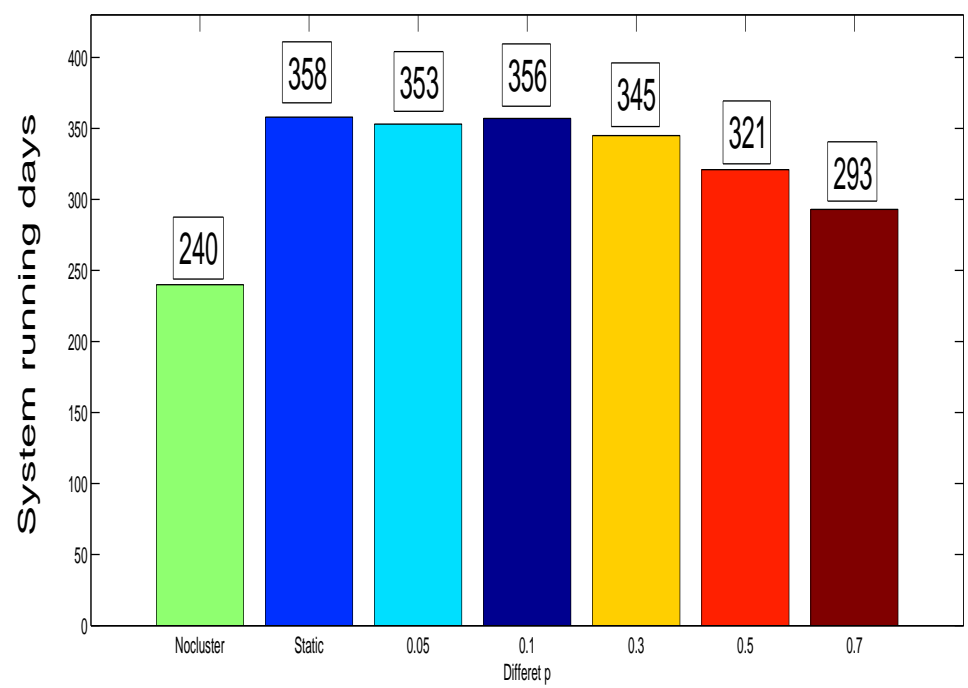

FIGURE 5.4: lifetime time. 


\section{Chapter 6}

\section{Dynamic Cluster Design}

So far, we have already introduced the static cluster design which can be used in the situation where we don't know the location of the devices, at the initial time, we can obtain the number of cluster heads when we only know how many devices will be deployed in the system. But if we have the devices positions at the initial time, could we find another way to make the optimal $p$ more accurate? The answer is yes. In this chapter we will describe the dynamic cluster design, which is based on the devices locations. By the end of this chapter, we will compare the dynamic design with static design, discussing the appropriate application conditions for them.

Referring to Figure (6.1), there are seven steps in our simulation. At the beginning, the M2M devices are deployed in a cell as in the static design. The position and initial energy of devices are also known. As we know, for the static cluster design, first we obtain an approximate optimal number of cluster heads and then form clusters based on this approximate number. But for the dynamic cluster design, since we know the number of devices and their locations, we try different numbers of cluster heads and obtain different system energy consumptions. After comparing the different values of energy consumption, we find an optimal $p$ value to obtain the lowest system consumption. On the next part, we will describe the following procedure for the dynamic design. 


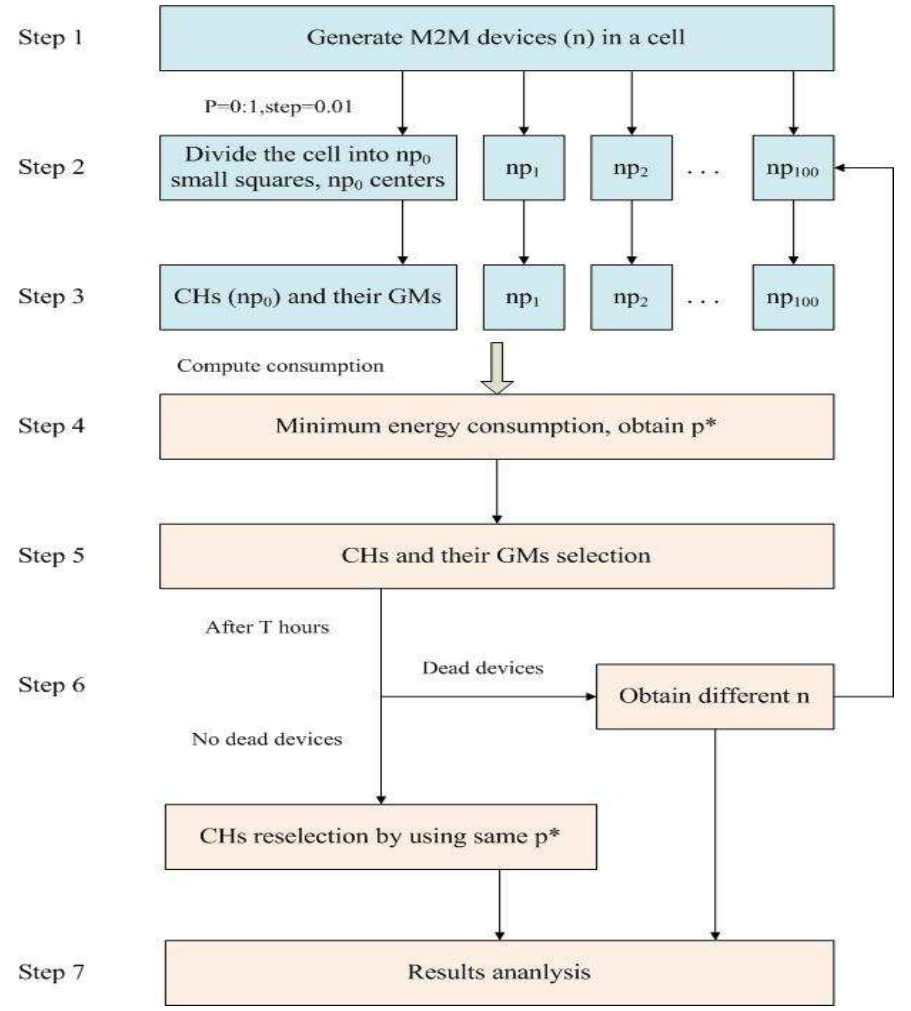

Figure 6.1: The flow chart for dynamic design.

\subsection{Cluster Form}

Described in Chapter 4, when the total number of devices are known, for each fixed value of $p$, we can obtain the number of cluster heads ( $n p)$. Moreover by using Algorithm (1), we divide the cell into $n p$ small squares. Since we know every device positions, based on different value of $p$, we divide the devices into different number of cluster group $(n p)$. Meanwhile it is easy to obtain each device cost function value by utilizing (4.8) and decide which devices will be cluster heads. Furthermore, Algorithm (2) is used for selecting group members.

So far, for the same number of devices (n), we can form different cluster groups in the cell, depended on different value of $p$. By using equation (6.1) (6.2) (6.3) and (6.4), we will figure out different system energy consumptions, based on different $p$. Because the locations of devices are known, we can decide which kind of pathloss will be utilized in 
intra communication.

$$
R_{J}^{d}(G M)= \begin{cases}\frac{w_{2}}{M_{K}+1} \log \left(1+\frac{P_{t}(G M)\left(M_{K}+1\right)}{N_{0} w_{2} 10 \frac{P L(a)_{J}+Z_{a}}{10}}\right), & D_{J}<r_{d} \\ \frac{w_{2}}{M_{K}+1} \log \left(1+\frac{P_{t}(G M)\left(M_{K}+1\right)}{N_{0} w_{2} 10 \frac{P L()_{J}+Z_{e}}{10}}\right), & D_{J}>r_{d}\end{cases}
$$

Where $R_{J}^{d}(G M)$ corresponds to the data rate for each group member, $D_{J}$ is the distance between cluster head and each group member. Therefor the consumption for every group member $\left(C_{J}^{d}(G M)\right)$ after sending one packet is

$$
C_{J}^{d}(G M)=\frac{P_{o}(G M) l}{R_{J}^{d}(G M)}+\frac{P_{w} l}{R_{J}^{d}(G M)}+P_{s}\left(t_{0}-\frac{l}{R_{J}^{d}(G M)}\right)
$$

Similar, the consumption for cluster heads $\left(C_{K}^{d}(C H)\right)$ is

$$
C_{K}^{d}(C H)=\frac{P_{o}(C H) \alpha\left(M_{K}+1\right) l}{R_{K}(C H)}+\frac{P_{w} \alpha\left(M_{K}+1\right) l}{R_{K}(C H)}+P_{s}\left(t_{0}-\frac{\alpha\left(M_{K}+1\right) l}{R_{K}(C H)}\right)
$$

Where the data rate for cluster head $\left(R_{K}(C H)\right)$ are obtain by (3.7), therefor whole system consumption is

$$
C^{d}(\text { system })=\sum_{K=1}^{n p}\left(C_{K}^{d}(C H)+\sum_{J=1}^{M_{K}} C_{K J}^{d}(G M)\right)
$$

Considering a certain number of devices $(n)$, we obtain each system consumption by using (6.4) as changing value of $p$. It is easy to find which value of $p$ has the smallest energy consumption. Therefor we will use this value $p$ as the optimal value to form the cluster groups at the initial time.

The optimal value of $p$ will change as the number of devices changing, We utilize Algorithm (4) to simulate the relationship between optimal $p$ and the number of M2M devices $n$. In Algorithm (4), based on different value $J$, we utilized the function Generate to deploy the devices. Then, we try different $p$ values with step size 0.01 to obtain the corresponding system consumption $C_{\text {system. }}$. If $p$ is 0 or 1 , we consider no clusters in the system. Function FindCloseDevice is used to find reference devices which are the blue points shown in Figure (4.3). Function ClusterDesign is used for forming the clusters and function Consumption is used for computing the system energy consumption. So, by using the result of Algorithm (4), we obtain Figure (6.2) 

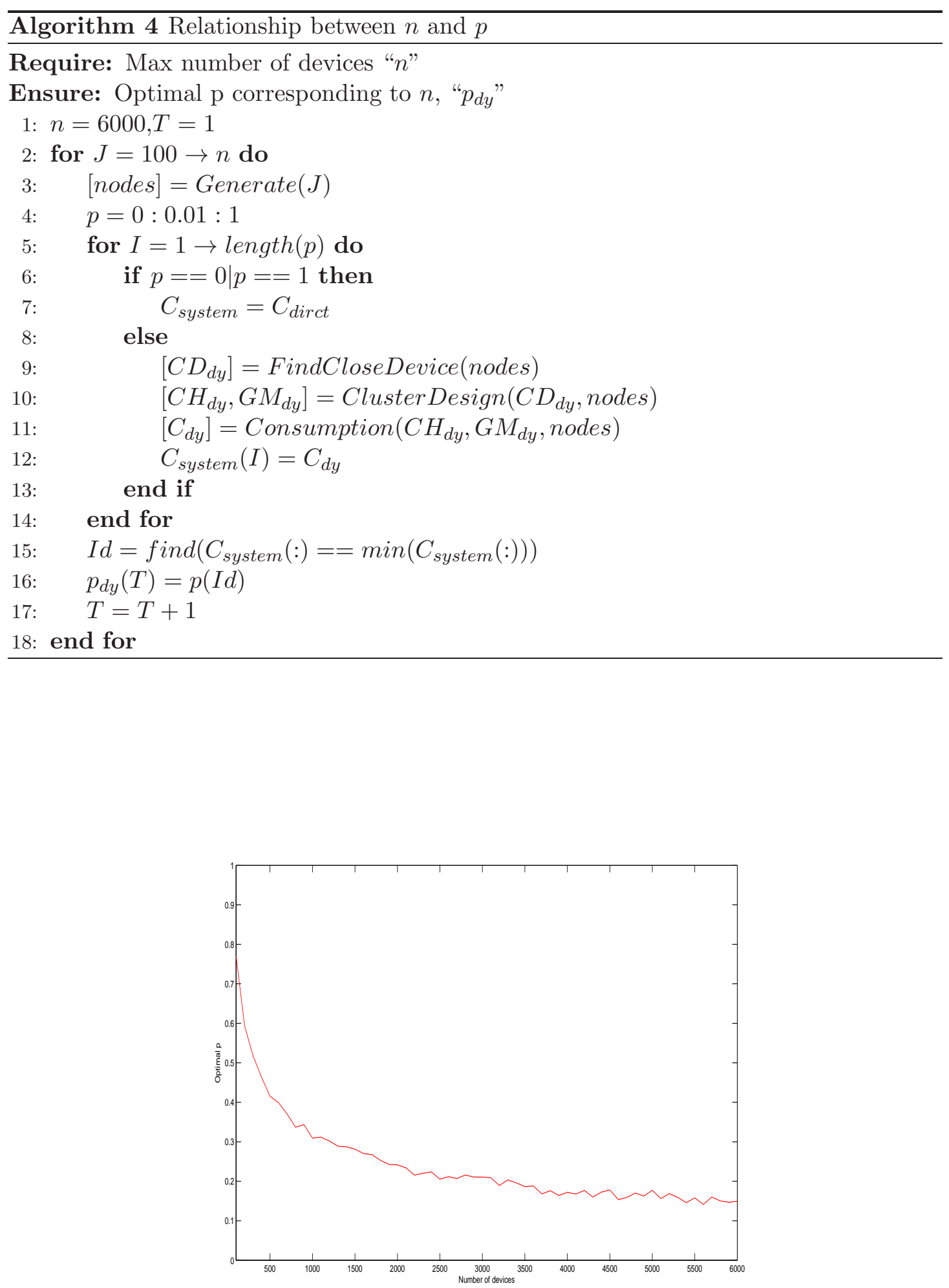

Figure 6.2: Optimal $p$ as different number in dynamic design. 
As we known, the dynamic cluster design utilizes the locations of the M2M devices to achieve the lowest energy consumption, so it should be more accurate than the static design. Based on Figure (6.2), when the number for devices is small $(n<300)$, the optimal $p$ is larger than 0.5 , which means that there is a high percentage of cluster heads in the system. As mentioned in the introduction, in assumption (7) in Chapter 3 , the devices which connect directly with the base station are named cluster heads, even if they don't have group members. When the number of devices in the cell is small, it means that the intensity of devices is small and the average distance among them is large. If the percentage of cluster heads is low, many group members need to use long distance propagation to transmit data to their cluster heads, then the cluster heads forward these accumulated data to the base station. This is not an energy efficient way in comparison to the case where the devices connect directly to the base station. Therefore, a high percentage of cluster heads will avoid long distance propagations for cluster group members and thus, save more energy.

As the devices increase, the value of the optimal $p$ value becomes smaller and smaller to finally reach a value between 0.1 and 0.2 . It means that for a large number of devices, the value of the optimal $p$ will not change significantly. Thus, based on the parameter used by us, the proportion of cluster heads and group members is suitable for saving energy.

\subsection{Cluster Reselection}

From above, we obtain an optimal number of cluster heads based on a certain number of M2M devices. Similar to the static cluster design, after T hours, the cluster heads will be reselected because we want to avoid certain devices to die faster than others. When changing the cluster heads, if the number of devices is constant, the reselection will be as in the static cluster design. If the number of devices is changed, such as after a long time of operation, some M2M devices will be dead and removed from the system, we will use a new number of total devices to repeat the step 2 to 5 in Figure (6.1), obtaining a new cluster heads number and form new cluster groups. Algorithm (5) describes the procedure of cluster reselection: 


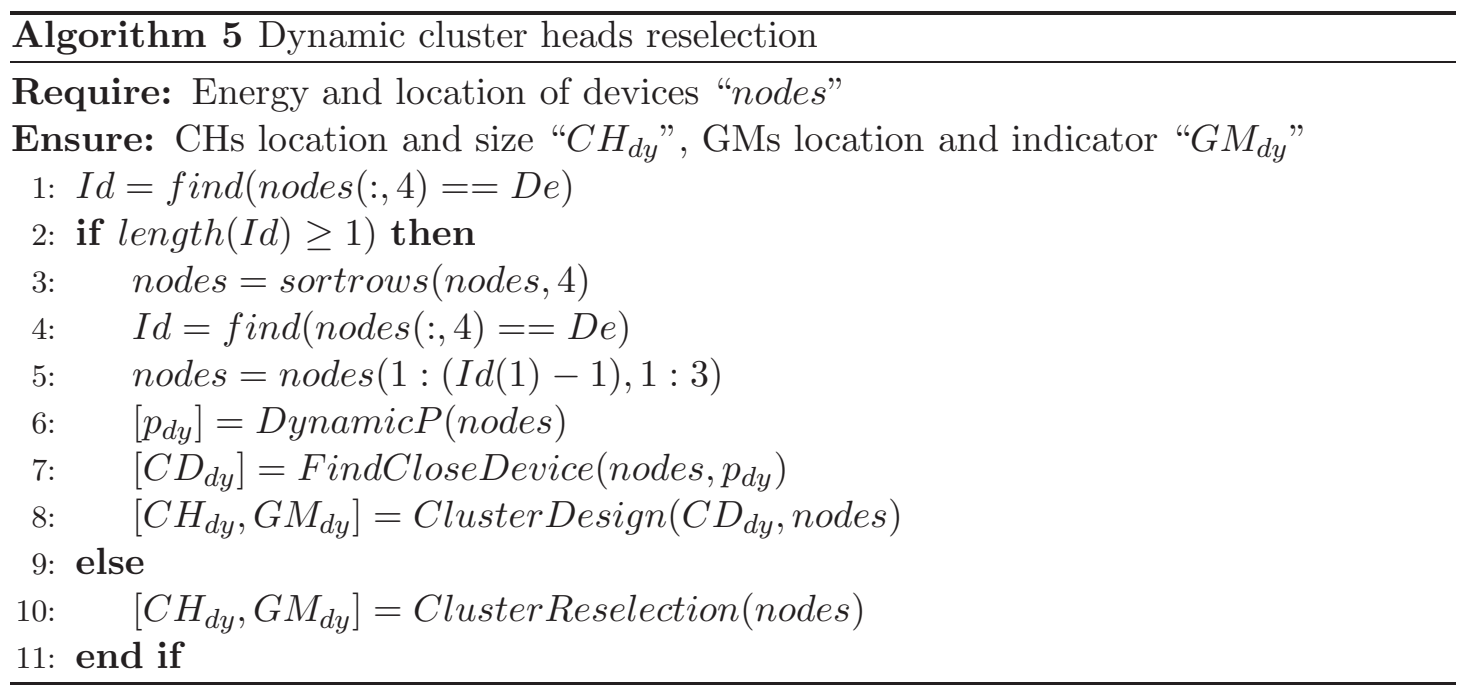

In here, nodes is a matrix which saves all the devices. It is a 4 columns matrix, where the first two columns are locations, the third one is residual energy and the final one is utilized for indicating dead devices (De means dead). DynamicP is the function which obtains the optimal $p$ value depending on different numbers of devices and their locations. ClusterReselection is used for reselecting the cluster heads and their group members depending on an unchanged optimal $p$.

\subsection{Result Comparing}

Based on the results of the dynamic cluster design and static cluster design using the same conditions, we want to obtain their different performances and draw some conclusions. Figure (6.3) shows different optimal $p$ values corresponding to different devices amounts when using these two designs.

In Figure (6.3) the red line is the dynamic design while blue line the static one. Since the static design uses the expectation function, the dynamic design should have a more accurate result. This conclusion can be proved in the following part. From the figure we can see that there is a large gap for the $p$ value between these two designs when the number of devices is less than 2000. The dynamic design has a higher percentage of cluster heads in respect to the static one, and the reason is not complicated. When the static design is used, we assume that all intra communication utilizes a short distance propagation model, but for the case when we have low intensity devices, the distance between GM and $\mathrm{CH}$ is longer than $r_{d}$ which is the actual boundary of distinction for 


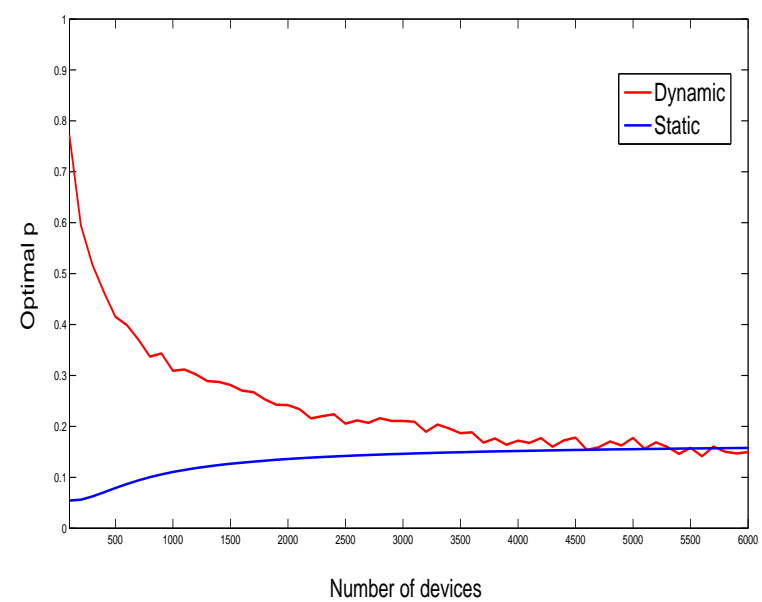

FiguRE 6.3: Optimal $p$ as different number by using dynamic and static design.

short and long distance propagation. The static design will select few cluster heads and other devices will connect to them, believing that the intra communication consumption is optimal and energy efficient. But the dynamic design will distinguish propagation models by distance, in the low intensity case, the distance for the intra communication is large so the dynamic design considers that more devices connecting directly to the BS is energy efficient. That is the reason why they have such gap for a low intensity situation. For a large number of devices, the optimal value of $p$ obtained by both designs is similar, meaning that the static design is more suitable for a deployment with large number of devices.

Figure (6.4) compares the whole system residual energy when using both designs for 500, 2000, 6000 devices during a simulation time of 30 days. The red line in Figure (6.4) is the dynamic design while the blue line is the static design. When $n$ is 500 , we can see how the dynamic design has a significant better performance in saving energy in comparison to the static design. When $\mathrm{n}$ is 2000 , the gap between these two designs is not obvious. For 6000 devices, they have almost the same performance when it comes to saving energy. Based on that figure, we can prove that the dynamic cluster design is better than the static design, but for a large number of devices, the static design is good enough for saving system energy.

Figure (6.5) compares the residual energy for 500, 2000, 6000 devices on $100^{\text {th }}, 150^{\text {th }}, 250^{\text {th }}$, $300^{\text {th }}$ day. Referring to Figure (6.5), it shows the same conclusion obtained from Figure 


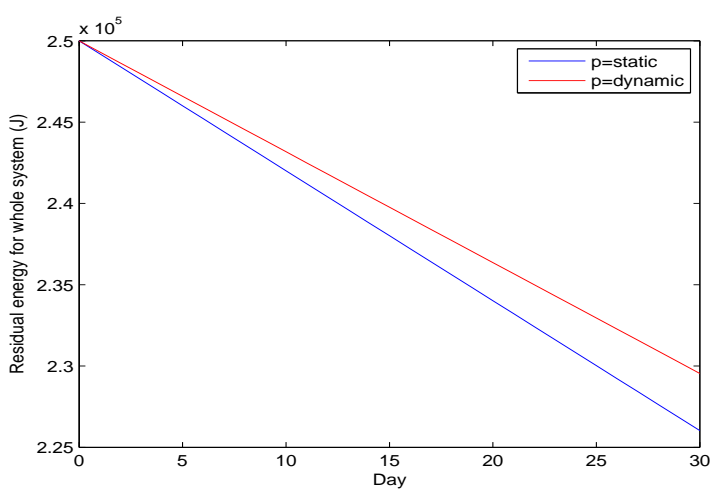

(a) $n=500$

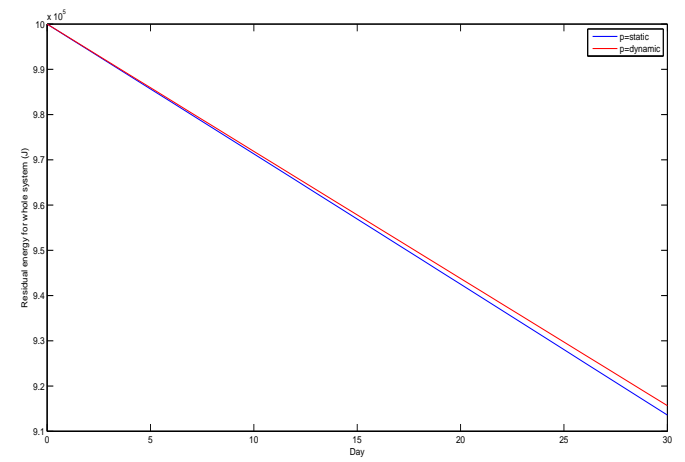

(b) $n=2000$

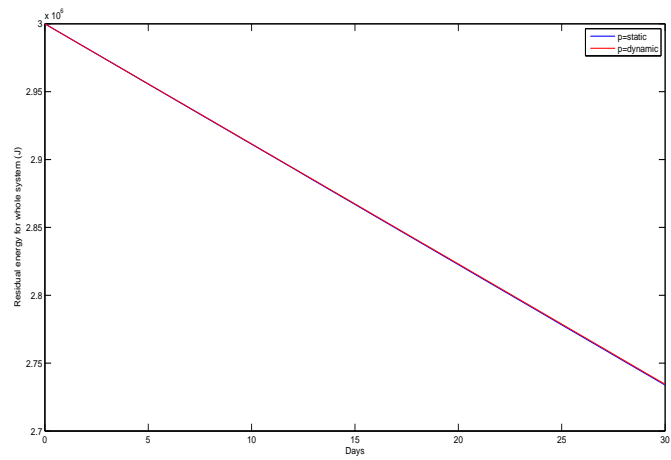

(c) $n=6000$

FIGURE 6.4: Residual energy comparing for different $n$ in 30 days 


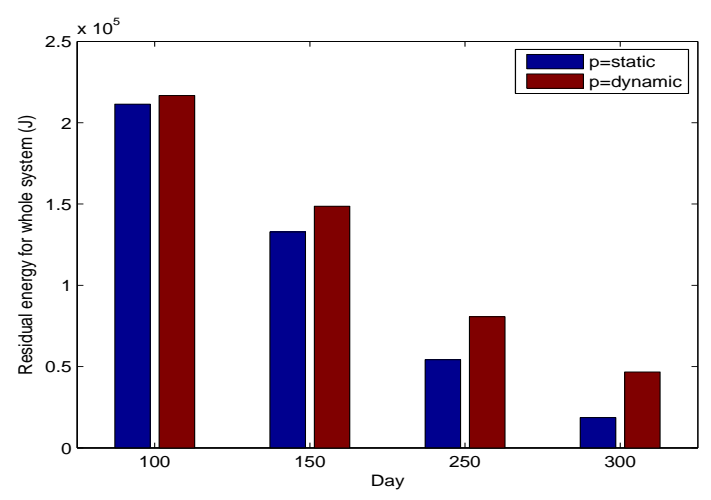

(a) $n=500$

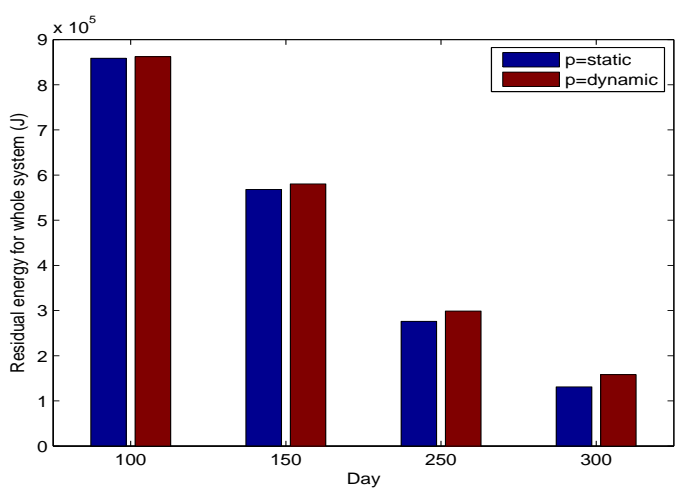

(b) $n=2000$

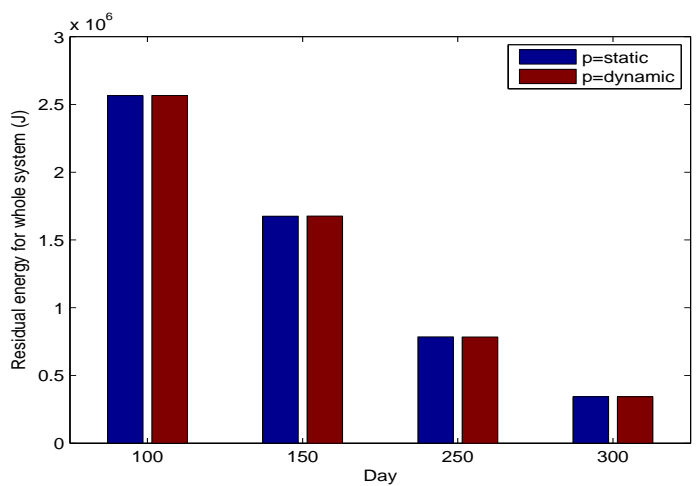

(c) $n=6000$

FIGURE 6.5: Residual energy comparing for different $n$ on $100^{\text {th }}, 150^{\text {th }}, 250^{\text {th }}, 300^{\text {th }}$ day. 


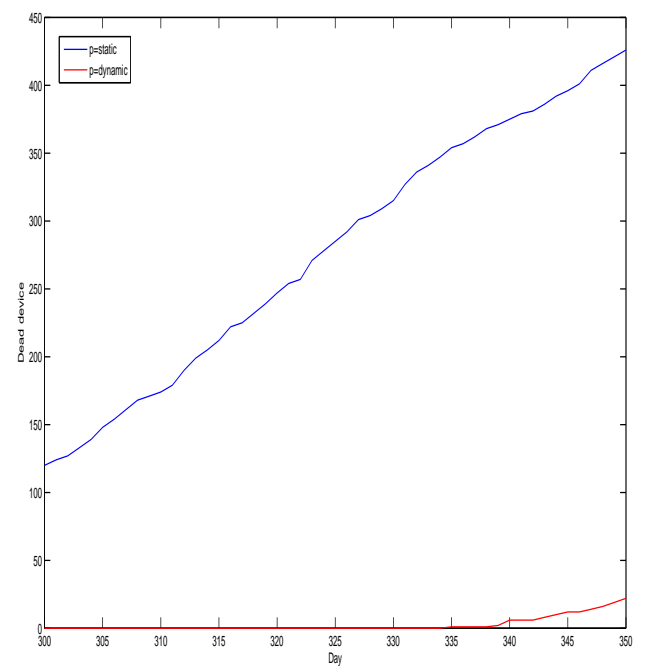

FIGURE 6.6: Dead devices for two ways with $n=500$.

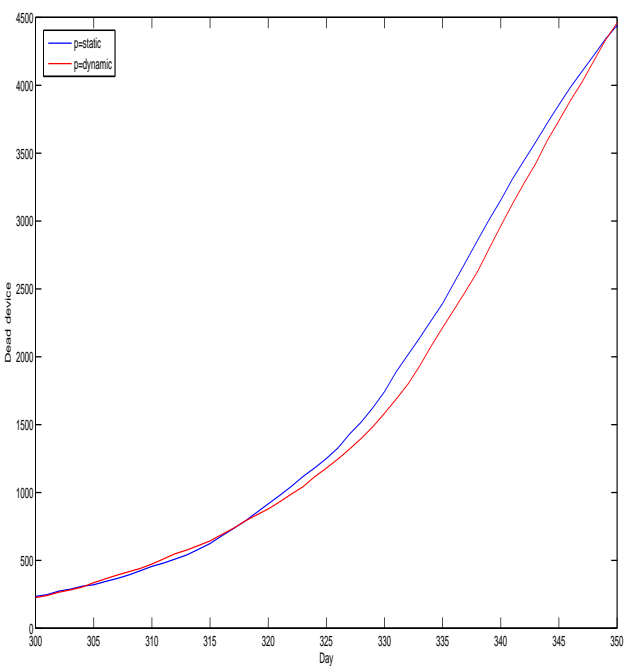

FIGURE 6.7: Dead devices for two ways with $n=6000$

(6.4) but in a longer period of time. The static cluster design is suitable for large number of devices in a cell and can save the energy almost like the dynamic cluster design does.

Figure (6.6) and (6.7) shows the comparison of dead devices. When $n$ is 500, the dynamic design (red line) has an absolutely good performance. When $n$ is 6000 , their performances are similar. 


\section{Chapter 7}

\section{Conclusion and Further Works}

Developments in the area of wireless communication allow the development of a new technology, M2M communications, which is used in several M2M applications [25]. M2M networks utilize multi-hop routing in order to arrange data routes in the wireless network. Therefore, the devices should be reliable by means of availability. A key point of longevity of these network is to have energy-efficient system architectures and algorithms.

In this report, we provide two energy efficiency cluster designs for M2M communications. The static cluster design has the advantages for fast deployment while reducing the computing complexity. Specifically, handling a large amount of M2M devices, the static design is able to obtain an appropriate number of cluster heads quickly, while offering a significant performance in terms of energy saving. But in the situation of low intensity devices, the performance of the static design is not as good as the dynamic design. The dynamic cluster design can operate very well and obtain good performance results not only in high intensity devices situation but also with low intensity devices, since it considers each device position and the actual energy consumption situation. As a trade off, it has higher complexity for computing the amount of clusters. Both designs improve the energy efficiency of M2M communications and reduce the whole system consumption in comparison to a non-cluster approach.

Although the cluster design implemented in the system is relatively elaborated, there is still some space for improvement. For example, when we design the channel model, we consider the aspect of different propagation models and shadow effects. There are still 
other factors which affect the channel model accuracy, such as different fading models and more complicate power controls. Another interest point is that we simulate the M2M system in one cell, which means that we don't consider interference with other cells. If in a further work interference can be included, it will make the simulation more practical and realistic.

Another challenge is that our designs are based on the network where devices are stationary. While it is true that most of today's M2M applications have few mobile devices in a network, it is likely that in the future there will be many M2M networks consisting of hundreds of mobile devices. In that case, the cluster design will need to face a trade off between computing complexity and fast implementation under dynamic topology conditions. Hence, M2M will continue inspiring research and the issue related to the energy efficiency will definitely be one of them. 


\section{Bibliography}

[1] P. Zhang, G. W. Miao, and J. M. Costa. Technical feasibility analysis of lte for m2m applications. December 2012.

[2] XiaoHang.Chen. Low-power mac design for $\mathrm{m} 2 \mathrm{~m}$ communications in cellular networks:protocols and algorithms. 2013.

[3] R. Ratasuk, J. Tan, and A. Ghosh. Coverage and capacity analysis for machine type communications in lte. pages 1-5, 2012. ISSN 1550-2252.

[4] Chieh-Yuan Ho and Ching-Yao Huang. Energy-saving massive access control and resource allocation schemes for $\mathrm{m} 2 \mathrm{~m}$ communications in ofdma cellular networks. Wireless Communications Letters, IEEE, 1(3):209-212, 2012. ISSN 2162-2337.

[5] Yan Zhang, Rong Yu, M. Nekovee, Yi Liu, Shengli Xie, and S. Gjessing. Cognitive machine-to-machine communications: visions and potentials for the smart grid. Network, IEEE, 26(3):6-13, 2012. ISSN 0890-8044.

[6] S. Abdul Salam, S.A. Mahmud, G. M. Khan, and H.S. Al-Raweshidy. M2m communication in smart grids: Implementation scenarios and performance analysis. In Wireless Communications and Networking Conference Workshops (WCNCW), 2012 IEEE, pages 142-147, 2012.

[7] M. Zubair Shafiq; Lusheng Ji; Alex X. Liu; Jeffrey Pang; Jia Wang. A first look at cellular machine-to-machine traffic: large scale measurement and characterization. Proceedings of the 12th ACM SIGMETRICS/PERFORMANCE joint international conference on Measurement and Modeling of Computer Systems, pages 65-76, 2012.

[8] Peng Cheng, Li Wang, Bin Zhen, and Shihua Wang. Feasibility study of applying lte to smart grid. In Smart Grid Modeling and Simulation (SGMS), 2011 IEEE First International Workshop on, pages 108-113, 2011. 
[9] Z. Fan and S. Tan. M2m communications for e-health:standards, enabling technologies, and research challenges. Medical Information and Communication Technology, pages 1-4, 2012.

[10] Sun-Jin Kim, Jung-Hae Seo, J. Krishna, and Sun-Joong Kim. Wireless sensor network based asset tracking service. In Management of Engineering Technology, 2008. PICMET 2008. Portland International Conference on, pages 2643-2647, 2008.

[11] 3GPP. System improvement for machine type communication. TR, (23.888), December 2012.

[12] 3GPPTR. Service improvement for machine type communication. (22.368), December 2012.

[13] TS102. Machine to machine communication m2m service requirements. (689), December 2012.

[14] S. Nirmala and R. Nallusamy. An energy-efficient and enhanced qos aware of mac application specific protocol in the distributed wireless sensor networks. In Advanced Computing (ICoAC), 2012 Fourth International Conference on, pages 1-4, 2012.

[15] Guowang Miao, N. Himayat, G.Y. Li, and S. Talwar. Low-complexity energyefficient scheduling for uplink ofdma. Communications, IEEE Transactions on, 60 (1):112-120, 2012. ISSN 0090-6778.

[16] N. Tekbiyik and E. U. Biyikoglu. Energy efficient wireless unicast routing alternatives for machine-to-machine networks. Network and Computer Applications, 34: 1587-1614, 2011.

[17] A.S.K. Mammu, A. Sharma, U. Hernandez-Jayo, and N. Sainz. A novel clusterbased energy efficient routing in wireless sensor networks. In Advanced Information Networking and Applications (AINA), 2013 IEEE 27th International Conference on, pages 41-47, 2013.

[18] S. Bandyopadhyay and E.J. Coyle. An energy efficient hierarchical clustering algorithm for wireless sensor networks. 3:1713-1723 vol.3, 2003. ISSN 0743-166X.

[19] O. Younis and Sonia Fahmy. Heed: a hybrid, energy-efficient, distributed clustering approach for ad hoc sensor networks. Mobile Computing, IEEE Transactions on, 3 (4):366-379, 2004. ISSN 1536-1233. 
[20] A. Chandrakasan W. Heinzelman and H. Balakrishnan. Energy-efficient communication protocol for wireless micro sensor networks. 33rd Hawaii International Conference on System Sciences, 2(10), 2000.

[21] Kab Seok Ko, Min Jeong Kim, Kuk Yeol Bae, Dan Keun Sung, Jae Heung Kim, and Jae Young Ahn. A novel random access for fixed-location machine-to-machine communications in ofdma based systems. Communications Letters, IEEE, 16(9): 1428-1431, 2012. ISSN 1089-7798.

[22] Kan Zheng, Fanglong Hu, Wenbo Wang, Wei Xiang, and M Dohler. Radio resource allocation in lte-advanced cellular networks with $\mathrm{m} 2 \mathrm{~m}$ communications. Communications Magazine, IEEE, 50(7):184-192, 2012. ISSN 0163-6804.

[23] S. G. Foss and S. A. Zuyev. On a voronoi aggregative process related to a bivariate poisson process. In Adv. in Appl. Probab, pages 965-981, 1996.

[24] Heinzelman W.B.; Chandrakasan A.P.; Balakrishnan H. An application-specific protocol architecture for wireless microsensor networks. Wireless Communications, 1(4):660-670, 2002.

[25] Seoksoo Kim Sungmo Jung, Jong Hyun Kim. Network modeling and energyefficiency optimization for advanced machine-to-machine sensor networks. Sensors, (12):14851-14861, 2012. ISSN 1424-8220. 
TRITA-ICT-EX-2013:250 